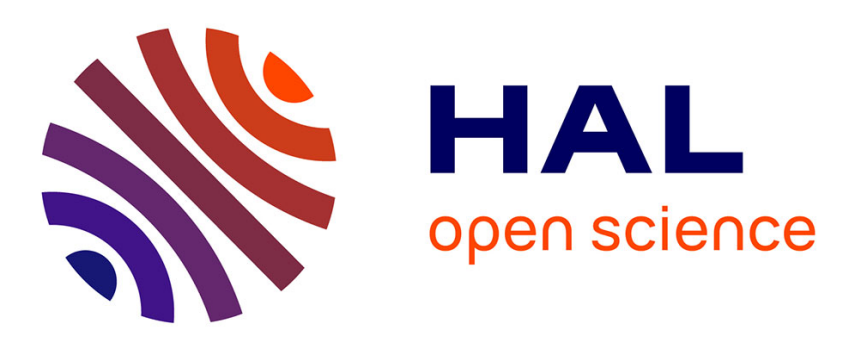

\title{
A new path to the non blow-up of incompressible flows Léo Agélas
}

\section{To cite this version:}

Léo Agélas. A new path to the non blow-up of incompressible flows. 2018. hal-01380349v4

\section{HAL Id: hal-01380349 \\ https://hal.science/hal-01380349v4}

Preprint submitted on 22 Jan 2019

HAL is a multi-disciplinary open access archive for the deposit and dissemination of scientific research documents, whether they are published or not. The documents may come from teaching and research institutions in France or abroad, or from public or private research centers.
L'archive ouverte pluridisciplinaire HAL, est destinée au dépôt et à la diffusion de documents scientifiques de niveau recherche, publiés ou non, émanant des établissements d'enseignement et de recherche français ou étrangers, des laboratoires publics ou privés. 


\title{
A new path to the non blow-up of incompressible flows
}

\author{
Léo Agélas \\ Department of Mathematics, IFP Energies Nouvelles, 1-4, avenue de Bois-Préau, F-92852 \\ Rueil-Malmaison, France
}

\begin{abstract}
One of the most challenging questions in fluid dynamics is whether the threedimensional (3D) incompressible Navier-Stokes, 3D Euler and two-dimensional Quasi-Geostrophic (2D QG) equations can develop a finite-time singularity from smooth initial data. Recently, from a numerical point of view, Luo \& Hou presented a class of potentially singular solutions to the Euler equations in a fluid with solid boundary Luo and Hou (2014a,b). Furthermore, in two recent papers Tao (2016a,b), Tao indicates a significant barrier to establishing global regularity for the 3D Euler and Navier-Stokes equations, in that any method for achieving this, must use the finer geometric structure of these equations. In this paper, we show that the singularity discovered by Luo \& Hou which lies right on the boundary is not relevant in the case of the whole domain $\mathbb{R}^{3}$. We reveal also that the translation and rotation invariance present in the Euler, Navier-Stokes and 2D QG equations are the key for the non blow-up in finite time of the solutions. The translation and rotation invariance of these equations combined with the anisotropic structure of regions of high vorticity allowed to establish a new geometric non blow-up criterion which yield us to the non blow-up of the solutions in all the Kerr's numerical experiments and to show that the potential mechanism of blow-up introduced in Brenner et al. (2016) cannot lead to the blow-up in finite time of solutions of Euler equations.
\end{abstract}

\section{Contents}

1 Introduction 2

2 Some notations and definitions $\quad 8$

3 Local regularity of the solutions 9

3.1 Local regularity for 3D Navier-Stokes or 3D Euler equations . . 10

3.2 Local regularity for $2 \mathrm{D}$ QG equation . . . . . . . . . . . 11

4 Assumption on the maximum vorticity

Email address: leo.agelas@ifpen.fr (Léo Agélas) 


\section{No blow up in finite time for numerical experiments}

7 Toward the non blowup in finite time of the solutions 29

7.1 Lagrangian flow map, vortex lines and vortex tubes . . . . . . . . 29

7.2 Anisotropic structure for the improvement of non blow-up criteria 31

\section{1. Introduction}

The Navier-Stokes and Euler equations describe the motion of a fluid in the three-dimensional space. These fundamental equations were derived over 250 years ago by Euler and since then have played a major role in fluid dynamics. They have enriched many branches of mathematics, were involved in many areas outside mathematical activity from weather prediction to exploding supernova (see for instance the surveys Constantin (2007),Bardos and Titi (2007)) and present important open physical and mathematical problems (see Constantin (2007)). Regarding the 2D Quasi-Geostrophic (2D QG) equation, it appears in atmospheric studies. It describes the evolution of potential temperature $u$ on the two dimensional boundary of a rapidly rotating half space with small Rossby and Ekman numbers, for the case of special solutions with constant potential vorticity in the interior and constant buoyancy frequency (normalized to one), where equations in the bulk are compressible Euler or Navier-Stokes equations coupled with temperature equation, continuity equation, and equation of state.

In the case of Navier-Stokes equations, for a long time ago, a global weak solution $u \in L^{\infty}\left(0, \infty ; L^{2}\left(\mathbb{R}^{3}\right)\right)^{3}$ and $\nabla u \in L^{2}\left(\mathbb{R}^{3} \times(0, \infty)\right)^{3}$ was built by Leray Leray (1934). In particular, Leray introduced a notion of weak solutions for the Navier-Stokes equations, and proved that, for every given $u_{0} \in L^{2}\left(\mathbb{R}^{3}\right)^{3}$, there exists a global weak solution $u \in L^{\infty}\left(\left[0,+\infty\left[; L^{2}\left(\mathbb{R}^{3}\right)\right)^{3} \cap L^{2}\left(\left[0, \infty\left[; \dot{H}^{1}\left(\mathbb{R}^{3}\right)\right)^{3}\right.\right.\right.\right.$. Hopf has proved the existence of a global weak solution in the general case $\mathbb{R}^{d}$, $d \geq 2$, Hopf (1951). Meanwhile the regularity and the uniqueness of this weak solution has been known for a long time ago for the two-dimensional case (see Ladyzhenskaya (1969), Lions and Prodi (1959), Lions (1969), Temam (1977)), in the three-dimensional case the problem remains widely open in spite of great efforts made. On the uniqueness many works have been done (see Furioli et al. (2000), Giga (1983), Monniaux (2000), Lions (1960), Gallagher and Planchon (2002)). Concerning the regularity of weak solutions, in Serrin (1962), it is proved that if $u$ is a Leray-Hopf weak solution belonging to $\left.L^{q}(10, T] ; L^{q}\left(\mathbb{R}^{3}\right)\right)^{3}$ with $\frac{2}{q}+\frac{3}{q} \leq 1,2<p<\infty, 3<q<\infty$, then the solution $\left.\left.u \in C^{\infty}\left(\mathbb{R}^{3} \times\right] 0, T\right]\right)^{3}$. In Von Wahl (1986) and Giga (1986), it is showed that if $u$ is a weak solution in $C\left([0, T] ; L^{3}\left(\mathbb{R}^{3}\right)\right)^{3}$, then $\left.\left.u \in C^{\infty}\left(\mathbb{R}^{3} \times\right] 0, T\right]\right)^{3}$. The limit case of $L^{\infty}\left([0, T] ; L^{3}\left(\mathbb{R}^{3}\right)\right)^{3}$ has been solved in Iskauriaza et al. (2003). Other criterion regularity can also be found in He (2002); Heywood (1988); Giga (1983); Kato (1984, 1990); Beirão da Veiga (1995); Chae and Choe (1999); Zhou (2002); Constantin and Fefferman (1994). 
In the case of Euler Equations, in the two dimension case, uniqueness and existence of classical solutions have been known for a long time ago (see Yudovich (1963, 1995); Vishik (1999); DiPerna and Majda (1987); Ladyzhenskaya (1969)). However for the full three space dimensions, little is known about smooth solutions apart from classical short-time existence and uniqueness. Moreover, weak solutions are known to be badly behaved from the point of view of Hadamard's well-posedness theory (see for instance the surveys De Lellis and Székelyhidi (2012); Villani (2008-2009)). Considerable efforts have been devoted to the study of the regularity properties of the 3D Euler equations. The main difficulty in the analysis lies in the presence of the nonlinear vortex stretching term and the lack of a regularization mechanism. Despite these difficulties, a few important partial results concerning the regularity of 3D Euler equations have been obtained over the years (see Beale et al. (1984); Ponce (1985); Ferrari (1993); Shirota and Yanagisawa (1993); Constantin et al. (1996); Deng et al. (2005); Gibbon and Titi (2013)).

In the case of 2D QG equation, besides its direct physical significance Held et al. (1995); Pedlosky (1987), the 2D QG equation has very interesting features of resemblance to the 3D Euler equation, being also an outstanding open problem of the finite time blow-up issue. In particular, one can derive a necessary and sufficient blow-up condition for the 2D QG equation similar to the well-known BealeKato-Majda (BKM) criterion (Beale-Kato-Majda Beale et al. (1984)). More precisely, the solution to the 2D QG equation (11) becomes singular at time $T^{*}$ if and only if $\int_{0}^{T^{*}}\left\|\nabla^{\perp} u(t)\right\|_{L^{\infty}} d t=+\infty$ (see Constantin et al. (1994)). Thus, $\nabla^{\perp} u$ plays a role similar to the vorticity $\omega$ in the 3D Euler equations. In the recent years, the $2 \mathrm{D}$ QG equation has been the focus of intense mathematical research Constantin et al. (1994); Córdoba (1998); Córdoba and Fefferman (2002); Chae (2003); Ohkitani and Yamada (1997); Constantin et al. (2012); Chae et al. (2012).

Unfortunately despite of considerable efforts devoted to the regularity issue of the 3D Euler, 3D Navier-Stokes and 2D QG equations, standard scaling heuristics have long indicated to the experts that the identity energy, together with the harmonic analysis estimates available for the heat equation and for the Euler bilinear operator, are not sufficient by themselves if one wishes to improve the theory on the Cauchy problem for these equations. It seems crucial to use the specific structure of the nonlinear term in these equations, as well as the divergence free assumption. Indeed, some finite time blowup results have been established for various Navier-Stokes type equations (see Montgomery-Smith (2001); Gallagher and Paicu (2009); Li and Sinai (2008); Plecháç and Sverák (2003); Katz and Pavlovic (2005)). Nevertheless, for all of these Navier-Stokes type equations, the cancellation property of the Euler bilinear operator did not hold and for some, the energy identity did not hold (see Montgomery-Smith (2001); Gallagher and Paicu (2009); Li and Sinai (2008)). 
However, recently it was shown also in Tao (2016a), a finite time blow up solution to an averaged three-dimensional Navier-Stokes equations of type $\partial_{t} u=$ $\Delta u+\tilde{B}(u, u)$, where $\tilde{B}$ is an averaged version of the Euler bilinear operator $B$, acting also on divergence free vector fields $u$ and obeying as $B$ to the cancellation property $\langle\tilde{B}(u, u), u\rangle=0$. This result suggests that any successful method to affirmatively answer to the Existence and Smoothness problem must either use finer structure of $B$ or else must rely crucially on some estimate or other property of the Euler bilinear operator $B$ that is not shared by the averaged operator $\tilde{B}$. Such additional structure exists for instance, the Euler equation has a vorticity formulation involving only differential operators rather than pseudo-differential ones.

However, even this vorticity formulation is not a barrier to get a finite time blow up solution. Indeed, it was shown in Tao (2016b), finite time blow-up solutions in the class of generalised Euler equations sharing with the Euler equation its main features such as: vorticity formulation, energy conservation, Kelvin circulation theorem, vorticity-vector potential formulation viewed as the Generalised Biot-Savart, function space estimates for the vector potential operator. Then, it seems that there is no room left to establish global regularity of solutions of 3D Euler equations. However, as it is mentioned in Tao (2016b), there are two properties of the Euler equations which are not obeyed by the generalised Euler equations, namely translation invariance and rotation invariance. Further, these symmetries basically determined the usual Biot-Savart law (see Kambe (2003a,b)) which are thus not shared by the Generalised Euler equations introduced in Tao (2016b). Furthermore, as it was shown in Constantin (1994); Constantin and Fefferman (1994), the use of Biot-Savart law yield to rewrite the vorticity equation in the case of Euler $(\nu=0)$ and Navier-Stokes $(\nu>0)$ equations as follows:

$$
\partial_{t} \omega+(\boldsymbol{v} \cdot \nabla) \omega-\nu \Delta \omega=\alpha \omega,
$$

where

$$
\alpha(x, t)=\frac{3}{4 \pi} P . V \cdot \int_{\mathbb{R}^{3}}(\hat{y} \cdot \xi(x, t)) \operatorname{det}(\hat{y}, \xi(x+y, t), \xi(x, t))|\omega(x+y, t)| \frac{d y}{|y|^{3}},
$$

with $\hat{y}=\frac{y}{|y|}, \xi=\frac{\omega}{|\omega|}$ and $\operatorname{det}(a, b, c)$ is the determinant of the matrix with columns $a, b, c$ in that order. We thus notice from the expression of $\alpha$ that if the direction of the vorticity, $\xi$ varies mildly within a small region around $x$, then the singularity of the integrand in (2) will be mild.

In this paper, we bring new insights which shed light on the mechanisms involved in the non blow-up of the solutions. We highlight through new geometric non blow-up criteria how the geometric regularity of the direction of vorticity combined with the anisotropic structure of the localized regions containing the positions where the maximum of the magnitude of the vorticity are reached, should prevent the formation of singularities. The novelty in the results of this paper lies on the use of the these two features in obtain- 
ing geometric non blow-up criteria using the finer structure of the Euler bilinear operator $B$. Up to now, many progress had been made to better take into account the geometrical properties and flow structures in the non blow-up criteria (see e.g Constantin and Fefferman (1994); Beirão da Veiga and Berselli (2009); Berselli (2009); Constantin (1994); Constantin et al. (1996); Hou and Li (2008); Deng et al. (2005, 2006a,b)). However none of these non blow-up criteria integrated both the geometric regularity of the direction of vorticity and the anisotropic structure of localized regions containing the positions where the maximum of the magnitude of the vorticity are reached. The most advanced non blow-up criteria were given in Deng et al. (2005, 2006a,b) and were established by using the Lagrangian formulation of the vorticity equation of the 3D Euler and 2D QG equations.

However the results obtained in Tao (2016b) suggest that even the most advanced non blow-up criterion Deng et al. (2005, 2006a) do not capture the finest structures of the the Euler bilinear operator $B$ since it was shown in Tao (2016b) that there exist generalised Euler equations sharing the same property than Euler equations as the Lagrangian formulation for their vorticity equations and for which their solutions blow up in finite time. Indeed, from Deng et al. (2005, 2006a), one can observe that the Deng-Hou-Yu non-blowup criterion can be applied to all the class of generalised Euler equations introduced in Tao (2016b). Then, in order to bring new insights in the investigation of whether the 3D incompressible Navier-Stokes, Euler and 2D QG equations can develop a finitetime singularity from smooth initial data, it was crucial to establish new non blow-up criteria which take into account the special structure of these equations not shared by the Generalised Euler equations.

Then in our Theorem 7.1, under mild assumptions based on the anisotropic structure of regions of high vorticity, we show that the solutions of 3D Euler, 3D Navier-Stokes and 2D QG equations cannot blow up at a finite time $T^{*}$ if

$$
\int_{0}^{T^{*}} \mathbf{A}_{d}(t)\left(1+\log ^{+}\left(\frac{\|\omega(t)\|_{\infty}}{\Omega(t)}\right)\right) d t<\infty
$$

where the functions $\mathbf{A}_{d}$ and $\Omega$ satisfy:

$$
\begin{aligned}
\mathbf{A}_{d}(t) & \leq\|\nabla \xi(t)\|_{\infty} \\
\Omega(t) & =\frac{\left(T^{*}-t\right)^{-1}}{1+\log ^{+}\left(\left(T^{*}-t\right)\|u(t)\|_{\infty} \mathbf{A}_{0}(t)\right)} \\
\mathbf{A}_{0}(t) & \leq\|\nabla \xi(t)\|_{\infty} .
\end{aligned}
$$

Note that $\xi(t)$ is well defined only on $\mathcal{O}(t)$ the set of points $x$ of $\mathbb{R}^{d}$ where $\omega(x, t) \neq 0$ and then $\|\nabla \xi(t)\|_{\infty}$ must be understood as $\|\nabla \xi(t)\|_{L^{\infty}(\mathcal{O}(t))}$.

In the case of 3D Euler equations and 2D QG equations by using their Lagrangian formulation, in Theorem 7.2 we go further in the non blow-up criteria by showing under mild assumptions based on the anisotropic structure of regions of high vorticity, that their solutions do not blow up at a finite time $T^{*}$ 


$$
\int_{0}^{T^{*}} \mathbf{A}_{d}(t) d t<\infty
$$

These results are obtained after a fine analysis of the term $\alpha$ defined by (2) combined with some results based on the anisotropic structure of regions of high vorticity. Our analysis starts by considering at each time $t \in] 0, T^{*}[$ the regions containing the positions where the maximum of the magnitude of the vorticity are reached and shrinking to zero as time tends to $T^{*}$ the alleged time of singularity. More precisely, these regions are balls of radius $\rho_{0}(t)=O\left(\left(T^{*}-t\right)\|u(t)\|_{\infty}\right)$ and of center the position of a point where the maximum of the magnitude of the vorticity is reached. Inside these regions, we then consider the regions of high vorticity for which the magnitude of the vorticity is greater than some function $\Omega(t)$ such that $\Omega(t) \gtrsim \frac{\left(T^{*}-t\right)^{-1}}{1+\log ^{+}\left(\rho_{0}(t)\|\nabla \xi(t)\|_{\infty}\right)}$.

In our analysis, to track in time the positions where the maximum of the magnitude of the vorticity is reached, we had to overcome the obstruction that we do not know if there exists an isolated absolute maximum for the vorticity achieved along a smooth curve in time as it was assumed in Proposition 2.1 of Constantin et al. (1994) and also in Deng et al. (2005, 2006a,b) (which assume that the position where the maximum of vorticity is reached, is advected with the flow). Moreover, recent numerical experiments show that it is not always the case (see Kerr and Bustamante (2012), see also section 5.4.5 in Grafke (2012)). We thus overcome this difficulty by using a result of Pshenichnyi concerning directional derivatives of the function of maximum and the structure of a set of supporting functionals Pshenichny (1971).

Our analysis led first to the non blow-up criterion given by our Theorem 5.1, namely, the solutions of 3D Euler, 3D Navier-Stokes and 2D QG equations cannot blow up at a finite time $T^{*}$ if

$$
\int_{0}^{T^{*}} \mathbf{A}_{d}(t) \pi(t) d t<\infty
$$

where the function $\pi$ is given by:

$$
\pi(t) \stackrel{\text { def }}{=} \sup _{x \in \Theta(t)} \sup _{0<R \leq \rho_{0}(t)} \frac{1}{R^{d-1}} \int_{B(x, R) \cap \mathcal{V}(t)}|\omega(z, t)| d z,
$$

182

with $\Theta(t) \stackrel{\text { def }}{=}\left\{x \in \mathbb{R}^{d} ;|\omega(x, t)|=\|\omega(t)\|_{\infty}\right\}$ and $\mathcal{V}(t) \stackrel{\text { def }}{=}\left\{z \in \mathbb{R}^{d} ;|\omega(z, t)| \geq\right.$ $\Omega(t)\}$.

In our Lemma 6.1, we thus derive a straightforward estimate of the function $\pi(t)$, that is

$$
\pi(t) \leq 3\|\omega(t)\|_{\infty} \sup _{x \in \Theta(t)}\left|\mathcal{V}(t) \cap B\left(x, \rho_{0}(t)\right)\right|^{\frac{1}{d}} .
$$

Thanks to the non blow-up criterion (3) and (5), we show the non blow-up in finite time of the solutions of Euler equations for Kerr's numerical experiments Kerr $(2005,1998,1997)$ without additional numerical tests as it was the 
case in Deng et al. (2005, 2006a), just by using the anisotropic structure of regions of high vorticity whose the features are described in Kerr (2005, 1998, 1997). Moreover, we show that the potential mechanism of blow-up introduced in Brenner et al. (2016) cannot lead to blow-up in finite time for Euler equations. To go further in our estimate of the function $\pi$, we use some assumptions characterizing the anisotropic structure of regions of high vorticity whose the justifications are given at the begininig of subsection 7.2 and we show in Proposition 7.1 that

$$
\pi(t) \lesssim 1+\log ^{+}\left(\frac{\|\omega(t)\|_{\infty}}{\Omega(t)}\right)
$$

which yield to Theorem 7.1.

In the case of Euler equations and 2D QG equations by using their Lagrangian formulation, after a fine and sharp analysis of the expression of the function $\pi$ (4) led thanks to our Lemmata 7.2,7.3 and 7.4, in Proposition 7.2 we go further in the non blow-up criteria by showing under mild assumptions based on the anisotropic structure of regions of high vorticity, that

$$
\pi(t)=O(1)
$$

We emphasize that according the 'thickness' of the structure of regions of high vorticity that these two estimates (6) and (7) can be much better. Indeed from the analysis led in Kuznetsov and Ruban (2000); Kuznetsov et al. (2001) for the study of collapse of vortex lines and agrees with numerical experiments Agafontsev et al. (2015, 2017), we could expect that (see Remark 7.1)

$$
\pi(t) \lesssim \Omega(t)^{-\frac{1}{2}}
$$

and then obtain in this case, the non blow-up in finite time of the solutions of Euler equations if

$$
\int_{0}^{T^{*}} \mathbf{A}_{d}(t) \Omega(t)^{-\frac{1}{2}} d t<\infty .
$$

We point out also that our geometric non blow-up criterion reveals the role of the geometric structures of the Incompressible flows in the non blow-up in finite time of the solutions and presents the advantage to be established in an Eulerian setting in comparison with all the recent geometric non blow-up criteria Constantin et al. (1996); Deng et al. (2005, 2006a); Constantin et al. (1994) using the Lagrangian formulation of Incompressible Inviscid Flows, which requires much more computational effort as it is mentioned in Grafke and Grauer (2013) and in section 5.4.5 of Grafke (2012). Furthermore, due to the existence of hyperbolic-saddle singularities suggested by the generation of strong fronts in geophysical/meteorology observations (see Constantin et al. (1994); Córdoba (1998)), and antiparallel vortex line pairing observed in numerical simulations and physical experiments, it was important to take them into account in our geometric non blow-up criterion. This is performed thanks to the term $\mathbf{D}_{d}(\hat{y}, \xi(x+y, t), \xi(x, t))$ (see $\left.(27),(28)\right)$ involved in the definition of the function $\mathbf{A}_{d}$ given at (39).

Then, the paper is organized as follows: 
- In section 2 , we give some notations and definitions.

- In section 3, we recall some results about the local regularity of solutions of Navier-Stokes, Euler and 2D QG equations.

- In section 4 , we give the reason for which we can assume for any time $t$ that $\|\omega(t)\|_{\infty}>0$ without loss of generality.

- In section 5, in Theorem 5.1, we establish a new geometric criterion for the non blow-up in finite time of the solutions of 3D Navier-Stokes, 3D Euler and 2D QG equations. We show that their solutions cannot blow up at a finite time $T^{*}$ if $\int_{0}^{T^{*}} \mathbf{A}_{d}(t) \pi(t) d t<\infty$, where $\mathbf{A}_{d}(t)$ is based on the regularity of the direction of the vorticity $\xi$ in regions shrinking to zero as time tends to $T^{*}$ and containing the positions where the maximum of the magnitude of the vorticity is reached (see definition of $\mathbf{A}_{d}$ at (39)).

- In section 6, we show the non-blowup in finite time of the solutions of the Euler equations in the numerical experiments considered these last years, by using inequality (5) about the function $\pi$ (4) and the anisotropic structure of regions of high vorticity described in Kerr $(2005,1998,1997)$.

- In section 7, we show the estimates (5), (6) and (7) concerning the function $\pi$ defined by (4), and obtain new non blow-up criteria in Theorems 7.1 and 7.2 .

Let us now introduce the 3D Navier-Stokes and Euler equations given by,

$$
\left\{\begin{array}{l}
\frac{\partial u}{\partial t}+(u \cdot \nabla) u+\nabla p-\nu \Delta u=0 \\
\nabla \cdot u=0
\end{array}\right.
$$

in which $u=u(x, t)=\left(u_{1}(x, t), u_{2}(x, t), u_{3}(x, t)\right) \in \mathbb{R}^{3}, p=p(x, t) \in \mathbb{R}$ and $\nu \geq 0$ ( $\nu=0$ corresponds to the Euler equations) denote respectively the unknown velocity field, the scalar pressure function of the fluid at the point $(x, t) \in \mathbb{R}^{3} \times[0, \infty[$ and the viscosity of the fluid,

with initial conditions,

$$
u(x, 0)=u_{0}(x) \text { for a.e } x \in \mathbb{R}^{3},
$$

where the initial data $u_{0}$ is a divergence free vector field on $\mathbb{R}^{3}$.

Regarding the $2 \mathrm{D}$ QG equation in $\mathbb{R}^{2}$, it is given by

$$
\left\{\begin{array}{l}
\frac{\partial u}{\partial t}+v \cdot \nabla u=0 \\
v=\nabla^{\perp}(-\Delta)^{-\frac{1}{2}} u
\end{array}\right.
$$

with initial data,

$$
u(x, 0)=u_{0}
$$


Here $\nabla^{\perp}=\left(-\partial_{x_{2}}, \partial_{x_{1}}\right)$. For $v$ we have also the following representation

$$
v=R^{\perp} u,
$$

where we have used the notation, $R^{\perp} u=\left(-R_{2} u, R_{1} u\right)$ with $R_{j}, j=1,2$, for the 2D Riesz transform defined by (see e.g. Stein (1970))

$$
R_{j}(u)(x, t)=\frac{1}{2 \pi} P . V \int_{\mathbb{R}^{2}} \frac{\left(x_{j}-y_{j}\right)}{|x-y|^{3}} u(y, t) d y .
$$

\section{Some notations and definitions}

In this section, we assume that $d \in \mathbb{N}, d \geq 2$.

For any vector $x=\left(x_{1}, x_{2}, \ldots, x_{d}\right) \in \mathbb{R}^{d}$, we denote by $|x|$ the euclidean norm of $x$ given by $|x|=\sqrt{\sum_{i=1}^{d}\left|x_{i}\right|^{2}}$. For any $y \in \mathbb{R}^{d}, y \neq 0$, we denote by $\hat{y}$ the unit vector $\hat{y}=\frac{y}{|y|}$. For any $m$-dimensional subset $A$ of $\mathbb{R}^{d}, 1 \leq m \leq d$, we denote by $|A|$ its measure. We denote by $\mathcal{M}\left(\mathbb{R}^{d}\right)$ the set of real square matrices of size $d$. We denote by Id the identity matrix of $\mathcal{M}\left(\mathbb{R}^{d}\right)$. For any vector field $v$ defined from $\mathbb{R}^{d}$ to $\mathbb{R}^{d}$, we denote by $\nabla v$ the gradient matrix of $v$, the matrix of $\mathcal{M}\left(\mathbb{R}^{d}\right)$ with $i j$-component, $\frac{\partial v_{i}}{\partial x_{j}}$ for all $1 \leq i, j \leq d$. For any real $a$, we denote by $a^{+}$ the real defined by $a^{+} \stackrel{\text { def }}{=} \max (a, 0)$. For any function $\varphi$ defined on $\mathbb{R}^{d} \times[0,+\infty[$, for all $t \geq 0$, we denote by $\varphi(t)$ the function defined on $\mathbb{R}^{d}$ by $x \longmapsto \varphi(x, t)$. We denote by $C_{c}^{\infty}\left(\mathbb{R}^{d}\right)$ the space of infinitely differentiable functions with compact support in $\mathbb{R}^{d}$. We denote by $B C$ the class of bounded and continuous functions and by $B C^{m}$ the class of bounded and $m$ times continuously derivable functions. For any $R>0$ and $x_{0} \in \mathbb{R}^{d}$, we denote by $B\left(x_{0}, R\right)$, the ball of $\mathbb{R}^{d}$ of center $x_{0}$ and radius $R$. For any $R>0$, we denote by $B_{R}$, the ball of $\mathbb{R}^{d}$ of center 0 and radius $R$.

We denote by div the differential operator given by, div $=\sum_{i=1}^{d} \frac{\partial}{\partial x_{i}}$.

We denote $A \lesssim B, B \gtrsim A$ or $A=O(B)$ the estimate $A \leq c B$ where $c>0$ is an absolute constant. If we need $c$ to depend on a parameter, we shall indicate this by subscripts, thus for instance $A \lesssim_{s}$ denotes the estimate $A \leq c_{s} B$ for some $c_{s}$ depending on $s$. We use $A \sim B$ as shorthand for $A \lesssim B \lesssim A$.

For any $f \in L^{p}\left(\mathbb{R}^{d}\right)$ (resp. $L^{p}\left(\mathbb{R}^{d}\right)^{d}$ or $L^{p}\left(\mathbb{R}^{d}\right)^{d \times d}$ ) with $1 \leq p \leq+\infty$, we denote by $\|f\|_{p}$ and $\|f\|_{L^{p}}$, the $L^{p}$-norm of $f$.

We denote by $H^{s}\left(\mathbb{R}^{d}\right)$ the Sobolev space $J^{-s} L^{2}\left(\mathbb{R}^{d}\right)$ where $J=(1-\Delta)^{\frac{1}{2}}$. We denote by $H_{\sigma}^{s}\left(\mathbb{R}^{3}\right)$ the Sobolev space $H_{\sigma}^{s}\left(\mathbb{R}^{3}\right) \stackrel{\text { def }}{=}\left\{\psi \in H^{s}\left(\mathbb{R}^{3}\right)^{3}: \operatorname{div} \psi=0\right\}$. In order to unify our notations with the two dimensional case $2 \mathrm{D}$ QG, we denote by $H_{\sigma}^{s}\left(\mathbb{R}^{2}\right)$ the Sobolev space $H^{s}\left(\mathbb{R}^{2}\right)$.

We denote by $\mathbb{P}$ the well-known $3 \mathrm{D}$ matrix Leray's projection operator with components, 


$$
\mathbb{P}_{i, j}=\delta_{i, j}-\frac{\partial}{\partial x_{i}} \frac{\partial}{\partial x_{j}} \Delta^{-1}=\delta_{i, j}-R_{j} R_{k}
$$

${ }_{289}$ where $R_{j}$ are the Riesz transform given by $R_{j}=\frac{\partial}{\partial x_{j}}(-\Delta)^{-\frac{1}{2}}=\frac{1}{4 \pi} \frac{x_{j}}{|x|^{4}} \star$ (see

290 Stein (1970) for more details), $\Delta^{-1}$ is the inverse of Laplace operator given by $\Delta^{-1}=-\frac{1}{4 \pi|x|} \star$, with $\star$ the convolution operator.

\section{Local regularity of the solutions}

In this section, we deal with the main result on local regularity of 3D Navier294 Stokes and Euler equations in its general form. By introducing $\mathbb{P}$ the matrix 295 Leray operator, Euler equations (9)-(10) can be re-written as follows,

$$
\frac{\partial u}{\partial t}+\mathbb{P}(u \cdot \nabla) u=0
$$

296 with initial conditions,

$$
u(0)=u_{0} .
$$

${ }_{297}$ For $u$ solution of (15)-(16), $\omega=\nabla \times u$ the vorticity of $u$ formally satisfies the 298 vorticity equation,

$$
\frac{\partial \omega}{\partial t}+(u \cdot \nabla) \omega-(\omega \cdot \nabla) u-\nu \Delta \omega=0,
$$

299 with initial conditions,

$$
\omega(0)=\omega_{0},
$$

300 where $\omega_{0}=\nabla \times u_{0}$ is the vorticity of $u_{0}$.

301 In the case of $2 \mathrm{D}$ QG equation, we get for $u$ solution of (11), $\omega=\nabla^{\perp} u$ the 302 vorticity of $u$ formally satisfies the vorticity equation,

$$
\frac{\partial \omega}{\partial t}+(v \cdot \nabla) \omega-(\omega \cdot \nabla) v=0
$$

303 with initial conditions,

$$
\omega(0)=\omega_{0},
$$

304 where $\omega_{0}=\nabla^{\perp} u_{0}$ is the vorticity of $u_{0}$.

In the region where $|\omega|>0$, we define $\xi$ the direction of the vorticity by $\xi=\frac{\omega}{|\omega|}$. 
3.1. Local regularity for 3D Navier-Stokes or 3D Euler equations

Assuming $u_{0} \in H_{\sigma}^{r}\left(\mathbb{R}^{3}\right)$ with $r>\frac{5}{2}$, thanks to Theorem 3.5 in Kato and Ponce (1988), Theorem 1 in Bourguignon and Brezis (1974) (see also Theorem I in Kato and Ponce (1986) and the results obtained in Beale et al. (1984)), we deduce that there exists a time $T>0$ such that there exists an unique strong solution $u \in C\left(\left[0, T\left[, H_{\sigma}^{r}\left(\mathbb{R}^{3}\right)\right) \cap C^{1}\left(\left[0, T\left[, H_{\sigma}^{r-2}\left(\mathbb{R}^{3}\right)\right)\right.\right.\right.\right.$ to the Navier-Stokes or Euler equations (15)-(16) and the energy equality holds for $u$, that means for all $t \in[0, T[$,

$$
\|u(t)\|_{2}+2 \nu \int_{0}^{t}\|\nabla u(s)\|_{2}^{2} d s=\left\|u_{0}\right\|_{2} .
$$

Moreover, if $u \notin C\left([0, T], H_{\sigma}^{r}\left(\mathbb{R}^{3}\right)\right)$, then we get (see Beale et al. (1984); Kato and Ponce (1988); Kozono and Taniuchi (2000)),

$$
\int_{0}^{T}\|\omega(t)\|_{\infty} d t=+\infty
$$

Notice thanks to Remark 3.7 in Kato and Ponce (1988), in the case of Euler equations, we get in addition that $u \in C^{1}\left(\left[0, T\left[, H_{\sigma}^{r-1}\left(\mathbb{R}^{3}\right)\right)\right.\right.$. We retrieve the pressure $p$ from the velocity $u$ with the formula,

$$
p=-\Delta^{-1} \operatorname{div}((u \cdot \nabla) u) .
$$

Furthermore, we get the local estimate (21). Indeed, thanks to remark 4.4 in Kato and Ponce (1988), we get

$$
\|u(t)\|_{H^{r}} \leq \frac{\left\|u\left(t_{0}\right)\right\|_{H^{r}}}{1-c\left\|u\left(t_{0}\right)\right\|_{H^{r}}\left(t-t_{0}\right)} \text { with } t_{0}<t<T,
$$

provided that $1-c\left\|u\left(t_{0}\right)\right\|_{H^{r}}\left(t-t_{0}\right)>0$, where $c>0$ is a constant.

\subsection{Local regularity for $2 D Q G$ equation}

This subsection is devoted to the local well-posedness of the 2D QG equation with a characterization of the maximal time existence of strong solutions. By using the same arguments as the proof of Proposition 4.2 in Agélas (2016), we get that the $H^{s}$-norm of $u$ is controlled by the integral in time of the maximum magnitude of the vorticity of $u$. A such Proposition has been proved in Constantin et al. (1994) for any integer $s \geq 3$, but here we extend this result to all real $s>2$. This improvement is obtained by using the logarithmic Sobolev inequality proved in Kozono and Taniuchi (2000); Kato and Ponce (1988) which requires only that $s>2$ instead of using the one proved in Beale et al. (1984) as it is the case in Constantin et al. (1994) and which requires integer $s \geq 3$. Then by using the same arguments as the proof of Proposition 4.3 in Agélas (2016), we get the following result which gives an improvement in comparison with Theorem 2.1 in Constantin et al. (1994):

Assuming $u_{0} \in H^{r}\left(\mathbb{R}^{2}\right)$ with $r>2$, we get that there exists a time $T>0$ such that there exists an unique strong solution $u \in C\left(\left[0, T\left[, H^{r}\left(\mathbb{R}^{2}\right)\right)\right.\right.$ to the 
2D QG equation (11)-(12) and the energy equality holds for $u$, that means for all $p \in[2, \infty]$ and $t \in[0, T$,

$$
\|u(t)\|_{p}=\left\|u_{0}\right\|_{p} .
$$

Moreover, if $u \notin C\left([0, T], H^{r}\left(\mathbb{R}^{2}\right)\right)$, then

$$
\int_{0}^{T}\|\omega(t)\|_{L^{\infty}} d t=+\infty
$$

Owing to $u \in C\left(\left[0, T\left[, H^{r}\left(\mathbb{R}^{2}\right)\right)\right.\right.$ and thanks to Lemma X4 in Kato and Ponce (1988), from $2 \mathrm{D}$ QG (11), we get $u \in C^{1}\left(\left[0, T\left[, H^{r-1}\left(\mathbb{R}^{2}\right)\right)\right.\right.$.

Similarly as in (21), we have

$$
\|u(t)\|_{H^{r}} \leq \frac{\left\|u\left(t_{0}\right)\right\|_{H^{r}}}{1-c\left\|u\left(t_{0}\right)\right\|_{H^{r}}\left(t-t_{0}\right)} \text { for } t_{0}<t<T,
$$

provided that $1-c\left\|u\left(t_{0}\right)\right\|_{H^{r}}\left(t-t_{0}\right)>0$, where $c>0$ is a constant.

\section{Assumption on the maximum vorticity}

Let $d \in\{2,3\}, r>\frac{d}{2}+1$ and $u_{0} \in H_{\sigma}^{r}\left(\mathbb{R}^{d}\right)$. Let $T^{*}>0$ be such that there exists a unique strong solution $u$ to the 3D Navier-Stokes, 3D Euler or 2D QG equations (9)-(10) in the class

$$
u \in C\left(\left[0, T^{*}\left[; H_{\sigma}^{r}\left(\mathbb{R}^{d}\right)\right) \cap C^{1}\left(\left[0, T^{*}\left[; H^{r-2}\left(\mathbb{R}^{d}\right)\right) .\right.\right.\right.\right.
$$

Thanks to the results of the section 3 , a such time $T^{*}$ exists.

In this paper, we are concerned with the non blowup in finite time of the solutions $u$ at times such $T^{*}$. Then, without loss of generality, in the whole of this paper, we consider only times of existence $T^{*}$ such that for all $t \in\left[0, T^{*}[\right.$,

$$
\|\omega(t)\|_{\infty}>0
$$

Indeed, let us assume that there exists $t_{0} \in\left[0, T^{*}\left[\right.\right.$ such that $\left\|\omega\left(t_{0}\right)\right\|_{\infty}=0$.

In the case of $2 \mathrm{D}$ QG equations (11), we get that $\omega\left(t_{0}\right) \equiv 0$ and then $\nabla u\left(t_{0}\right) \equiv 0$. Since $x \mapsto u\left(t_{0}, x\right)$ vanishes at infinity, then we get $u\left(t_{0}\right) \equiv 0$. Then by using inequality (24) concerning the local regularity, we deduce that $u(t) \equiv 0$ for all $t \in\left[t_{0}, T^{*}\left[\right.\right.$ and no blowup can occur at the time $T^{*}$.

By following step by step the proof of Lemma 4 given in Deng et al. (2005) but keeping the term $\|u(t)\|_{L^{2}\left(\mathbb{R}^{d}\right)}$ after using the Cauchy-Schwarz inequality, we obtain for all $t \in\left[0, T^{*}[\right.$,

$$
\begin{aligned}
\|u(t)\|_{\infty} & \lesssim\|u(t)\|_{2}^{\frac{2}{d+2}}\|\omega(t)\|_{\infty}^{\frac{d}{d+2}} \\
& \leq\left\|u_{0}\right\|_{2}^{\frac{2}{d+2}}\|\omega(t)\|_{\infty}^{\frac{d}{d+2}}
\end{aligned}
$$

where we have used (19) for the last inequality. Then thanks to (26) used with $d=3$, we obtain that $\left\|u\left(t_{0}\right)\right\|_{\infty} \equiv 0$ which implies that $u\left(t_{0}\right) \equiv 0$. Then by using the inequality (21) of local regularity, we deduce $u(t) \equiv 0$ for all $t \in\left[t_{0}, T^{*}[\right.$ and thus no blowup can occur at the time $T^{*}$. 


\section{Geometric properties for non blow-up of the solutions}

Historically, non blow-up criteria for the incompressible Euler equations and 2D QG equations commonly focus on global features of the flow, such as norms of the velocity or the vorticity fields. This comes at the disadvantage of neglecting the structures and physical mechanisms of the flow evolution. A strategy for overcoming such shortcomings was established by focusing more on geometrical properties and flow structures (see e.g. Constantin et al. (1996); Cordoba and Fefferman (2001)), such as vortex tubes or vortex lines.

In particular, in Constantin et al. $(1996,1994)$ the authors showed that local geometric regularity of the unit vorticity vector can lead to depletion of the vortex stretching. They prove that if there is up to time $T$ an $O(1)$ region in which the vorticity vector is smoothly directed, i.e., the maximum norm of $\nabla \xi$ (here $\xi=\frac{\omega}{|\omega|}, \omega$ the vorticity) in this region is $L^{2}$ integrable in time from 0 to $T$, and the maximum norm of velocity in some $O(1)$ neighbourhood of this region is uniformly bounded in time, then no blow-up can occur in this region up to time $T$.

However, this theorem dealt with $O(1)$ regions in which the vorticity vector is assumed to have some regularity, while in numerical computations, the regions that have such regularity and contain maximum vorticity are all shrinking with time (see Kerr (1993, 1995, 1997, 1998); Pelz (1997, 2001)).

Inspired by the work of Constantin et al. $(1996,1994)$, in Deng et al. (2005, $2006 a, b)$ the authors showed that geometric regularity of Lagrangian vortex filaments, even in an extremely localized region containing the maximum of vorticity which may shrink with time, can lead to depletion of the nonlinear vortex stretching, thus avoiding finite time singularity formation of the 3D Euler equations and 2D QG equations.

However, all the recent geometric constraints for non blow-up criteria of Euler and 2D QG equations based on local geometric regularity of Lagrangian vortex filaments Deng et al. $(2005,2006 \mathrm{a}, \mathrm{b})$ make the assumption that the position where the maximum of vorticity is reached, is advected with the flow, however it is not always the case, as described in Kerr and Bustamante (2012) (see also section 5.4.5 of Grafke (2012)).

Then in our Theorem 5.1, we establish in an Eulerian setting a new geometric non blow-up criterion for the Navier-Stokes, Euler and 2D QG equations based on the regularity of the direction of the vorticity in extremely localized regions containing the positions where the maximum of the magnitude of the vorticity are reached and shrinking to zero as time increase to some $T^{*}$ the alleged time of singularity. Our Eulerian geometric non blow-up criterion should give also new impetus to the numerical experiments due to their ease of implementation in comparison with Lagrangian geometric non blow-up criteria (see Grafke and Grauer (2013), see also section 5.4.5 of Grafke (2012)). Moreover our geometric non blow-up criterion is also valid for the Navier-Stokes equations that is not the case for the existing geometric non blow-up criteria obtained in Constantin et al. (1996); Deng et al. (2005, 2006a,b) based on a Lagrangian for- 
mulation of Incompressible Inviscid Flows.

To obtain our Theorem 5.1, we begin with Lemma 5.1.

Lemma 5.1. Let $d \in \mathbb{N}^{*}, T>0$ and $f \in C\left([0, T] ; B C\left(\mathbb{R}^{d}\right)\right)$ such that $\inf _{t \in[0, T]}\|f(t)\|_{\infty}>0$ and for any $t \in[0, T],|f(x, t)| \rightarrow 0$ as $|x| \rightarrow+\infty$. Then there exists $R>0$ such that for all $t \in[0, T],\|f(t)\|_{\infty}=\sup _{x \in B_{R}}|f(x, t)|$.

Proof. We set $a=\inf _{t \in[0, T]}\|f(t)\|_{\infty}>0$. Since $t \mapsto f(t)$ is a continuous function from the compact $[0, T]$ into the metric space $L^{\infty}\left(\mathbb{R}^{d}\right)$ then it is uniformly continuous. Hence, there exists $N \in \mathbb{N}^{*}$ such that for all $t, t^{\prime} \in[0, T],\left|t-t^{\prime}\right| \leq \frac{T}{N}$ we have $\left\|f(t)-f\left(t^{\prime}\right)\right\|_{\infty} \leq \frac{a}{4}$. We introduce the subdivision $\left\{t_{i}\right\}_{\{i \in \llbracket 0, N \rrbracket\}}$ of $[0, T]$ defined by $t_{i}=i \frac{T}{N}$ for $i \in \llbracket 0, N \rrbracket$. Since for any $t \in[0, T],|f(x, t)| \rightarrow 0$ as $|x| \rightarrow+\infty$, then for each $i \in \llbracket 0, N \rrbracket$, there exists $R_{i}>0$ such that for all $|x| \geq R_{i},\left|f\left(x, t_{i}\right)\right| \leq \frac{a}{4}$. We set $R=\max _{i \in \llbracket 0, N \rrbracket} R_{i}$. Let $t \in[0, T]$ then there exists $j \in \llbracket 0, N \rrbracket$ such that $\left|t-t_{j}\right| \leq \frac{T}{N}$ and hence for all $|x| \geq R \geq R_{j}$, we have $|f(x, t)| \leq\left|f(x, t)-f\left(x, t_{j}\right)\right|+\left|f\left(x, t_{j}\right)\right| \leq \frac{a}{2} \leq \frac{\|f(t)\|_{\infty}}{2}$. Then, we infer that for all $t \in[0, T],\|f(t)\|_{\infty}=\sup _{x \in B_{R}}|f(x, t)|$, which concludes the proof.

Before to prove Theorem 5.1, we need to introduce the following function $\mathbf{D}_{d}$ defined from $\mathbb{R}^{d} \times \mathbb{R}^{d} \times \mathbb{R}^{d}$ to $\mathbb{R}$ with $d \in\{2,3\}$ as follows: for $d=3$,

$$
\mathbf{D}_{d}\left(a_{1}, a_{2}, a_{3}\right)=\left(a_{1} \cdot a_{3}\right) \operatorname{Det}\left(a_{1}, a_{2}, a_{3}\right) .
$$

The Det in $\mathbf{D}_{d}$ is the determinant of the matrix whose columns are the three unit column vectors $a_{1}, a_{2}, a_{3}$. We observe that $\operatorname{Det}\left(a_{1}, a_{2}, a_{3}\right)=a_{1} \cdot\left(a_{2} \times a_{3}\right)$, then, we get

$$
\mathbf{D}_{d}\left(a_{1}, a_{2}, a_{3}\right)=\left(a_{1} \cdot a_{3}\right) a_{1} \cdot\left(a_{2} \times a_{3}\right) .
$$

and for $d=2$,

$$
\mathbf{D}_{d}\left(a_{1}, a_{2}, a_{3}\right)=\left(a_{1} \cdot a_{3}^{\perp}\right)\left(a_{2} \cdot a_{3}^{\perp}\right),
$$

where for any $z=\left(z_{1}, z_{2}\right) \in \mathbb{R}^{2}, z^{\perp}=\left(-z_{2}, z_{1}\right)$. We can notice that for $d \in\{2,3\}$ the function $\mathbf{D}_{d}$ is linear from its second variable.

From (27) and (28) we get $\mathbf{D}_{d}\left(a_{1}, a_{3}, a_{3}\right)=0$ then we deduce that for any $a_{1}, a_{2}, a_{3} \in B(0,1)$,

$$
\left|\mathbf{D}_{d}\left(a_{1}, a_{2}, a_{3}\right)\right| \leq\left|a_{2}-a_{3}\right|
$$

and we get also

$$
\left|\mathbf{D}_{d}\left(a_{1}, a_{2}, a_{3}\right)\right| \leq 1 .
$$

Now, we turn to the proof of our Theorem.

Theorem 5.1. Let $d \in\{2,3\}, u_{0} \in H_{\sigma}^{r}\left(\mathbb{R}^{d}\right)$ with $r>\frac{d}{2}+3$. Let $T^{*}>0$ be such that there exists a unique strong solution $u$ to the $3 D$ Navier-Stokes, 3D Euler equations (9)-(10) or 2D QG equations (11)-(12) in the class

$$
u \in C\left(\left[0, T^{*}\left[; H_{\sigma}^{r}\left(\mathbb{R}^{d}\right)\right) \cap C^{1}\left(\left[0, T^{*}\left[; H^{r-2}\left(\mathbb{R}^{d}\right)\right) .\right.\right.\right.\right.
$$


${ }^{439}$ Let $\rho_{0}$ be the function defined from $\left[0, T^{*}[\right.$ to $] 0,+\infty\left[\right.$ for all $t \in\left[0, T^{*}[\right.$ by

$$
\rho_{0}(t) \stackrel{\text { def }}{=} 36\left(T^{*}-t\right)\|u(t)\|_{\infty} .
$$

${ }_{440}$ Let $\mathbf{A}_{0}$ be the function defined from $\left[0, T^{*}[\right.$ to $] 0,+\infty\left[\right.$ for all $t \in\left[0, T^{*}[\right.$ by:

$$
\mathbf{A}_{0}(t) \stackrel{\text { def }}{=} \sup _{x \in \Theta(t)} \sup _{y \in B\left(0, \rho_{0}(t)\right) \backslash\{0\}} \frac{\mathbf{D}_{d}(\hat{y}, \xi(x+y, t), \xi(x, t))^{+}}{|y|},
$$

${ }_{441}$ where for any $t \in\left[0, T^{*}[\right.$

$$
\Theta(t) \stackrel{\text { def }}{=}\left\{x \in \mathbb{R}^{d} ;|\omega(x, t)|=\|\omega(t)\|_{\infty}\right\} .
$$

${ }_{442}$ Let $\Omega$ be the function defined from $\left[0, T^{*}[\right.$ to $] 0,+\infty[$ by:

$$
\Omega(t) \stackrel{\text { def }}{=} \frac{\left(T^{*}-t\right)^{-1}}{8\left(1+\log ^{+}\left(4 \rho_{0}(t) \mathbf{A}_{0}(t)\right)\right)} .
$$

${ }_{443}$ We introduce also the set of high vorticity regions defined for all $t \in\left[0, T^{*}[\right.$ by

$$
\mathcal{V}(t) \stackrel{\text { def }}{=}\left\{z \in \mathbb{R}^{d} ;|\omega(z, t)| \geq \Omega(t)\right\} .
$$

${ }_{444}$ Let $\pi$ be the function defined from $\left[0, T^{*}\left[\right.\right.$ to $\left[0,+\infty\left[\right.\right.$, for all $t \in\left[0, T^{*}[\right.$ by

$$
\pi(t) \stackrel{\text { def }}{=} \sup _{x \in \Theta(t)} \sup _{0<R \leq \rho_{0}(t)} \frac{1}{R^{d-1}} \int_{B(x, R) \cap \mathcal{V}(t)}|\omega(z, t)| d z .
$$

${ }_{445}$ Let $\rho$ be the function defined from $\left[0, T^{*}\left[\right.\right.$ to $\left[0,+\infty\left[\right.\right.$ for all $t \in\left[0, T^{*}[\right.$ by

$$
\rho(t) \stackrel{\text { def }}{=} 4(d+1) c_{d}\left(T^{*}-t\right) \pi(t)
$$

${ }_{446}$ where $c_{d}=\frac{3}{4 \pi}$ if $d=3, c_{d}=\frac{1}{2 \pi}$ else.

${ }_{447}$ Let $\mathbf{A}_{d}$ be the function defined from $\left[0, T^{*}\left[\right.\right.$ to $\left[0,+\infty\left[\right.\right.$ for all $t \in\left[0, T^{*}[\right.$ by

$$
\mathbf{A}_{d}(t) \stackrel{\text { def }}{=} \sup _{x \in \Theta(t)} \sup _{y \in B(0, \rho(t)) \backslash\{0\}} \frac{\mathbf{D}_{d}(\hat{y}, \xi(x+y, t), \xi(x, t))^{+}}{|y|} .
$$

${ }_{448}$ Then if there exists $t_{1} \in\left[0, T^{*}[\right.$ such that

$$
\int_{t_{1}}^{T^{*}} \mathbf{A}_{d}(t) \pi(t)<+\infty
$$

${ }_{449}$ then the solution $u$ cannot blowup at the finite time $T^{*}$.

${ }_{450}$ Moreover, we have for all $t \in\left[0, T^{*}[\right.$ and $x \in \Theta(t)$,

$$
\nabla|\omega|(x, t)=0 \text { and } \nabla \cdot \xi(x, t)=0 .
$$


${ }_{451}$ Proof. Let $0<T<T^{*}$. We want first to apply Lemma 5.1 to the function $\omega$, ${ }_{452}$ then we check that the hypotheses of the Lemma are satisfied.

${ }_{453}$ Since $u \in C\left([0, T] ; H^{r}\left(\mathbb{R}^{d}\right)\right) \cap C^{1}\left([0, T] ; H^{r-2}\left(\mathbb{R}^{d}\right)\right)$, then we infer that $\omega \in$ $\left.\left.{ }_{454} C\left([0, T] ; H^{r-1}\left(\mathbb{R}^{d}\right)\right) \cap C^{1}(] 0, T\right] ; H^{r-3}\left(\mathbb{R}^{d}\right)\right)$. Thanks to the Sobolev embedding ${ }_{455} H^{s}\left(\mathbb{R}^{d}\right) \hookrightarrow B C^{m}\left(\mathbb{R}^{d}\right)$ for $s>\frac{d}{2}+m, m \in \mathbb{N}$ and since $r>\frac{d}{2}+3$ we deduce 456 that $\omega \in C\left([0, T] ; B C^{2}\left(\mathbb{R}^{d}\right)\right) \cap C^{1}\left([0, T] ; B C\left(\mathbb{R}^{d}\right)\right)$. Thanks to (25), we get ${ }_{457}$ that $\inf _{t \in[0, T]}\|\omega(t)\|_{\infty}>0$. Moreover, since $\omega \in C\left([0, T] ; H^{r-1}\left(\mathbb{R}^{d}\right)\right)$ with $r>$ ${ }_{458} \frac{d}{2}+3$, we have for any $t \in[0, T],|\omega(x, t)| \rightarrow 0$ as $|x| \rightarrow+\infty$, the proof follows 459 immediately by using the density of $C_{0}^{\infty}\left(\mathbb{R}^{d}\right)$ in $H^{r-1}\left(\mathbb{R}^{d}\right)$ and the Sobolev embedding $H^{r-1}\left(\mathbb{R}^{d}\right) \hookrightarrow L^{\infty}\left(\mathbb{R}^{d}\right)$ for $r>\frac{d}{2}+3$.

Then thanks to Lemma 5.1, there exists $R>0$ such that for all $t \in[0, T]$, $\|\omega(t)\|_{\infty}=\sup _{x \in B_{R}}|\omega(x, t)|$. Then for all $t \in[0, T]$, the set $\Theta(t)$ defined by (34) can be rewritten as follows:

$$
\Theta(t)=\left\{x \in B_{R} ;|\omega(x, t)|=\|\omega(t)\|_{\infty}\right\}
$$

We introduce the direction of the vorticity $\xi=\frac{\omega}{|\omega|}$ defined on the non empty open set $\mathcal{O} \stackrel{\text { def }}{=}\left\{(x, t) \in \mathbb{R}^{d} \times[0, T] ;|\omega(x, t)|>0\right\}$.

We set $\boldsymbol{v}=u$ in the case of 3D Navier-Stokes or 3D Euler equations and $\boldsymbol{v}=R^{\perp} u$ with $\nu=0$ in the case of $2 \mathrm{D}$ QG equation.

Then by multiplying (17) or (18) by $\xi$, we get that for all $(x, t) \in \mathcal{O}$,

$$
\begin{aligned}
\frac{\partial|\omega|}{\partial t}(x, t)+\boldsymbol{v}(x, t) \cdot \nabla|\omega|(x, t) & -(\omega(x, t) \cdot \nabla) \boldsymbol{v}(x, t) \cdot \xi(x, t) \\
& -\nu \Delta|\omega|(x, t)+\nu|\omega(x, t)||\nabla \xi(x, t)|^{2}=0 .
\end{aligned}
$$

We introduce the function $\varphi$ defined for all $t \in[0, T]$ by

$$
\varphi(t) \stackrel{\text { def }}{=} \sup _{x \in B_{R}}|\omega(x, t)|
$$

and we search the expression of its derivative. For this, we use the main Theorem obtained in Pshenichny (1971) or Theorem 1 in Borisenko and Minchenko (1992) after verifying that the hypotheses of the Theorem are satisfied.

Since $\omega \in C\left([0, T] ; B C^{2}\left(\mathbb{R}^{d}\right)\right) \cap C^{1}\left([0, T] ; B C\left(\mathbb{R}^{d}\right)\right)$, then we deduce that $|\omega| \in$ $B C(\mathcal{O}), \frac{\partial|\omega|}{\partial t} \in B C(\mathcal{O})$ and $\nabla^{2}|\omega| \in B C(\mathcal{O})$. Since for any $t \in[0, T], \Theta(t) \subset$ $\mathcal{O} \times\{t\}$, then, thanks to the results obtained in Pshenichny (1971) (see also Theorem 1 in Borisenko and Minchenko (1992)), by using also (41) we obtain the expression of the derivative of $\varphi$ given for any $t \in[0, T]$ by,

$$
\varphi^{\prime}(t)=\sup _{x \in \Theta(t)} \frac{\partial|\omega|}{\partial t}(x, t)
$$




$$
\begin{aligned}
\frac{\partial|\omega|}{\partial t}(x, t) & =\frac{\partial|\omega|}{\partial t}(x, t)+\boldsymbol{v}(x, t) \cdot \nabla|\omega|(x, t) \\
& =(\omega(x, t) \cdot \nabla) \boldsymbol{v}(x, t) \cdot \xi(x, t)+\nu \Delta|\omega|(x, t)-\nu|\omega(x, t)||\nabla \xi(x, t)|^{2} \\
& \leq(\omega(x, t) \cdot \nabla) \boldsymbol{v}(x, t) \cdot \xi(x, t),
\end{aligned}
$$

481 where we have used (42) for the second equality and (44) for the last inequality.

${ }_{482}$ We can notice that we get equality for (45) in the case of $3 \mathrm{D}$ Euler or 2D

${ }_{483} \mathrm{QG}$ equations, since for these equations we have not the terms $\nu \Delta|\omega|(x, t)$ and ${ }_{484} \nu|\omega(x, t)||\nabla \xi(x, t)|^{2}$.

485 Then using (45), from (43), we obtain,

$$
\varphi^{\prime}(t) \leq \sup _{x \in \Theta(t)}(\omega(x, t) \cdot \nabla) \boldsymbol{v}(x, t) \cdot \xi(x, t),
$$

${ }_{486}$ which means that

$$
\frac{d}{d t}\|\omega(t)\|_{\infty} \leq \sup _{x \in \Theta(t)}(\omega(x, t) \cdot \nabla) \boldsymbol{v}(x, t) \cdot \xi(x, t)
$$

${ }_{487}$ where equality holds in the case of 3D Euler or 2D QG equations. We use ${ }_{488}$ now the function $\alpha$ introduced in Constantin (1994); Constantin and Fefferman 489 (1994) for the 3D Navier-Stokes or 3D Euler equations and in Constantin et al. 490 (1994) for the 2D QG equation, defined for all $(x, t) \in \mathcal{O}$ by,

$$
\alpha(x, t)=c_{d} P . V \cdot \int_{\mathbb{R}^{d}} \mathbf{D}_{d}(\hat{y}, \xi(x+y, t), \xi(x, t))|\omega(x+y, t)| \frac{d y}{|y|^{d}},
$$

491 where $\hat{y}=\frac{y}{|y|}$ and in the case of 3D Navier-Stokes or 3D Euler equations for ${ }_{492}$ which $d=3, c_{d}=\frac{3}{4 \pi}$ and in the case of $2 \mathrm{D}$ QG equation for which $d=2$, ${ }_{493} \quad c_{d}=\frac{1}{2 \pi}$. We use the fact that $|\omega(x+y, t)| \xi(x+y, t)=\omega(x+y, t)$ and the ${ }_{494}$ fact that $\mathbf{D}_{d}$ is linear in comparison with its second variable, to rewrite (47) as 495 follows:

$$
\alpha(x, t)=c_{d} P . V \cdot \int_{\mathbb{R}^{d}} \mathbf{D}_{d}(\hat{y}, \omega(x+y, t), \xi(x, t)) \frac{d y}{|y|^{d}} .
$$

${ }_{496}$ By using the Biot-Savart law (see Chorin and Marsden (1993)) for which in the ${ }_{497}$ case of Euler and Navier-Stokes equations, we have

$$
\boldsymbol{v}(x, t)=\frac{1}{4 \pi} \int_{\mathbb{R}^{3}} \frac{y}{|y|^{3}} \times \omega(x+y) d y,
$$


${ }_{498}$ and in the case of $2 \mathrm{D}$ QG equations, we get an equivalent formula

$$
\boldsymbol{v}(x, t)=\frac{1}{2 \pi} \int_{\mathbb{R}^{2}} \frac{1}{|y|} \omega(x+y, t) d y,
$$

we deduce as in Constantin (1994); Constantin and Fefferman (1994) and Constantin et al.

500 (1994) that for all $(x, t) \in \mathcal{O}$

$$
(\omega(x, t) \cdot \nabla) \boldsymbol{v}(x, t) \cdot \xi(x, t)=\alpha(x, t)|\omega(x, t)| .
$$

501 Therefore, from (46), we deduce that for all $t \in[0, T]$,

$$
\begin{aligned}
\frac{d}{d t}\|\omega(t)\|_{\infty} & \leq \sup _{x \in \Theta(t)} \alpha(x, t)|\omega(x, t)| \\
& =\left(\sup _{x \in \Theta(t)}(\alpha(x, t))\|\omega(t)\|_{\infty},\right.
\end{aligned}
$$

${ }_{502}$ where we have used the fact that for all $x \in \Theta(t),|\omega(x, t)|=\|\omega(t)\|_{\infty}$. Let us estimate now $\alpha(x, t)$ for any $t \in[0, T]$ and $x \in \Theta(t)$. For this purpose, let us take $t \in[0, T]$ and $x \in \Theta(t)$, then we decompose the term $\alpha(x, t)$ as the sum of three terms,

$$
\alpha(x, t)=I_{1}+I_{2}+I_{3}
$$

506 where,

$$
I_{1}=c_{d} \int_{B\left(0, \min \left(\rho(t), \rho_{0}(t)\right)\right)} \mathbf{D}_{d}(\hat{y}, \omega(x+y, t), \xi(x, t)) \frac{d y}{|y|^{d}},
$$

507

$$
I_{2}=c_{d} \int_{B\left(0, \rho_{0}(t)\right) \cap B\left(0, \min \left(\rho(t), \rho_{0}(t)\right)\right)^{c}} \mathbf{D}_{d}(\hat{y}, \omega(x+y, t), \xi(x, t)) \frac{d y}{|y|^{d}}
$$

508 and

$$
I_{3}=c_{d} \int_{B\left(0, \rho_{0}(t)\right)^{c}} \mathbf{D}_{d}(\hat{y}, \omega(x+y, t), \xi(x, t)) \frac{d y}{|y|^{d}} .
$$

${ }_{509}$ Then, we estimate the three terms $I_{1}, I_{2}$ and $I_{3}$. For the term $I_{1}$, from (51) we 510 get

$$
\begin{aligned}
I_{1} & =c_{d} \int_{B\left(0, \min \left(\rho(t), \rho_{0}(t)\right)\right)} \frac{\mathbf{D}_{d}(\hat{y}, \xi(x+y, t), \xi(x, t))}{|y|}|\omega(x+y, t)| \frac{d y}{|y|^{d-1}} \\
& \leq c_{d} \mathbf{A}_{d}(t) \int_{B\left(0, \min \left(\rho(t), \rho_{0}(t)\right)\right)} \frac{|\omega(x+y, t)|}{|y|^{d-1}} d y \\
& =c_{d} \mathbf{A}_{d}(t) \int_{B\left(x, \min \left(\rho(t), \rho_{0}(t)\right)\right) \cap \mathcal{V}(t)^{c}} \frac{|\omega(z, t)|}{|x-z|^{d-1}} d z \\
& +c_{d} \mathbf{A}_{d}(t) \int_{\left.B\left(x, \min \left(\rho(t), \rho_{0}(t)\right)\right)\right) \cap \mathcal{V}(t)} \frac{|\omega(z, t)|}{|x-z|^{d-1}} d z \\
& \leq c_{d} \mathbf{A}_{d}(t) \Omega(t) \int_{B(0, \rho(t))} \frac{d y}{|y|^{d-1}}+c_{d} \mathbf{A}_{d}(t) \int_{\left.B\left(x, \min \left(\rho(t), \rho_{0}(t)\right)\right)\right) \cap \mathcal{V}(t)} \frac{|\omega(z, t)|}{|z-x|^{d-1}} d z .
\end{aligned}
$$


511 Furthermore, we have

$$
\int_{B(0, \rho(t))} \frac{d y}{|y|^{d-1}}=|B(0,1)| \rho(t) .
$$

${ }_{512}$ Therefore, by using the fact that $c_{d}|B(0,1)| \leq 1$ (since $|B(0,1)|=\frac{4 \pi}{3}$ for $d=3$

513 and $|B(0,1)|=\pi$ for $d=2)$, we deduce

$$
I_{1} \leq \mathbf{A}_{d}(t) \Omega(t) \rho(t)+\mathbf{A}_{d}(t) I_{1,1}
$$

514 where

$$
I_{1,1} \stackrel{\text { def }}{=} c_{d} \int_{B\left(x, \min \left(\rho(t), \rho_{0}(t)\right)\right) \cap \mathcal{V}(t)} \frac{|\omega(z, t)|}{|z-x|^{d-1}} d z .
$$

515 Let $\varepsilon(t) \stackrel{\text { def }}{=} \frac{\pi(t)}{\|\omega(t)\|_{\infty}}$, then we have

$$
I_{1,1} \leq c_{d} \int_{B(x, \varepsilon(t))} \frac{|\omega(z, t)|}{|z-x|^{d-1}} d z+c_{d} I_{1,2},
$$

516 with

$$
I_{1,2} \stackrel{\text { def }}{=} \int_{B(x, \varepsilon(t))^{c} \cap B\left(x, \min \left(\rho(t), \rho_{0}(t)\right)\right) \cap \mathcal{V}(t)} \frac{|\omega(z, t)|}{|z-x|^{d-1}} d z .
$$

517 On one hand, we have

$$
\begin{aligned}
c_{d} \int_{B(x, \varepsilon(t))} \frac{|\omega(z, t)|}{|z-x|^{d-1}} d z & \leq c_{d}\|\omega(t)\|_{\infty} \int_{B(x, \varepsilon(t))} \frac{d z}{|z-x|^{d-1}} \\
& =c_{d}|B(0,1)|\|\omega(t)\|_{\infty} \varepsilon(t) \\
& \leq \pi(t) .
\end{aligned}
$$

${ }_{518}$ On the other hand, we estimate $I_{1,2}$.

${ }_{519} \quad$ If $\mathcal{V}(t)=\emptyset$ or $\min \left(\rho(t), \rho_{0}(t)\right) \leq \varepsilon(t)$ then from (57) we infer that $I_{1,2}=0$.

${ }_{520}$ Let us assume now that $\mathcal{V}(t) \neq \emptyset$ and $\min \left(\rho(t), \rho_{0}(t)\right)>\varepsilon(t)$. Then, from $(57)$

521 we get

$$
\begin{aligned}
I_{1,2} & =\int_{\varepsilon(t)}^{\min \left(\rho(t), \rho_{0}(t)\right)}\left(\int_{\partial B(x, R) \cap \mathcal{V}(t)}|\omega(z, t)| \frac{d \gamma(z)}{|z-x|^{d-1}}\right) d R \\
& =\int_{\varepsilon(t)}^{\min \left(\rho(t), \rho_{0}(t)\right)} \frac{1}{R^{d-1}}\left(\int_{\partial B(x, R) \cap \mathcal{V}(t)}|\omega(z, t)| d \gamma(z)\right) d R .
\end{aligned}
$$

${ }_{522}$ We introduce the function $F_{t}$ defined from $\left[0, \rho_{0}(t)\right]$ to $[0,+\infty[$ for all $0 \leq R \leq$ ${ }_{523} \rho_{0}(t)$ by

$$
F_{t}(R) \stackrel{\text { def }}{=} \int_{B(x, R) \cap \mathcal{V}(t)}|\omega(z, t)| d z
$$


${ }_{524}$ We observe that $\frac{d F_{t}}{d R}(R)=\int_{\partial B(x, R) \cap \mathcal{V}(t)}|\omega(z, t)| d \gamma(z)$, then from (59) we get ${ }_{525} \quad I_{1,2}=\int_{\varepsilon(t)}^{\min \left(\rho(t), \rho_{0}(t)\right)} \frac{1}{R^{d-1}} \frac{d F_{t}}{d R}(R) d R$ and by using an integration by parts we 526 obtain

$$
I_{1,2}=\frac{F_{t}\left(\min \left(\rho(t), \rho_{0}(t)\right)\right)}{\min \left(\rho(t), \rho_{0}(t)\right)^{d-1}}-\frac{F_{t}(\varepsilon(t))}{\varepsilon(t)^{d-1}}+(d-1) \int_{\varepsilon(t)}^{\min \left(\rho(t), \rho_{0}(t)\right)} \frac{F_{t}(R)}{R^{d}} d R .
$$

${ }_{527}$ From (60), we notice that $\pi(t)=\sup _{0<R \leq \rho_{0}(t)} \frac{F_{t}(R)}{R^{d-1}}$, then from (61) we deduce

$$
\begin{aligned}
I_{1,2} & \leq \pi(t)+(d-1) \pi(t) \int_{\varepsilon(t)}^{\min \left(\rho(t), \rho_{0}(t)\right)} \frac{d R}{R} \\
& =\pi(t)\left(1+(d-1) \log \left(\frac{\min \left(\rho(t), \rho_{0}(t)\right)}{\varepsilon(t)}\right)\right) .
\end{aligned}
$$

${ }_{528}$ Therefore whatever the case we get

$$
I_{1,2} \leq \pi(t)\left(1+(d-1) \log ^{+}\left(\frac{\min \left(\rho(t), \rho_{0}(t)\right)}{\varepsilon(t)}\right)\right) .
$$

529 Since $\min \left(\rho(t), \rho_{0}(t)\right) \leq \rho(t)$ and thanks to (38) we get

$$
\begin{aligned}
\frac{\min \left(\rho(t), \rho_{0}(t)\right)}{\varepsilon(t)} & \leq 4(d+1) c_{d}\left(T^{*}-t\right)\|\omega(t)\|_{\infty} \\
& \leq 4\left(T^{*}-t\right)\|\omega(t)\|_{\infty} .
\end{aligned}
$$

530 Therefore from (62) we infer

$$
I_{1,2} \leq \pi(t)\left(1+(d-1) \log ^{+}\left(4\left(T^{*}-t\right)\|\omega(t)\|_{\infty}\right)\right) .
$$

${ }_{531}$ Thanks to (55)-(58) and (63), we get

$$
I_{1,1} \leq \pi(t)+c_{d} \pi(t)\left(1+(d-1) \log ^{+}\left(4\left(T^{*}-t\right)\|\omega(t)\|_{\infty}\right)\right) .
$$

532 Then thanks to (64), from (54) we obtain

$$
I_{1} \leq \mathbf{A}_{d}(t)\left(\Omega(t) \rho(t)+\pi(t)+c_{d} \pi(t)\left(1+(d-1) \log ^{+}\left(4\left(T^{*}-t\right)\|\omega(t)\|_{\infty}\right)\right)\right) .
$$

By using the definition of the functions $\rho$ and $\Omega$, for which we have $\Omega(t) \rho(t) \leq$ ${ }_{534} 4 c_{d}(d+1) \pi(t)$, from (65) we deduce

$$
\begin{aligned}
I_{1} & \leq \mathbf{A}_{d}(t) \pi(t)\left(4 c_{d}(d+1)+1+c_{d}\left(1+(d-1) \log ^{+}\left(4\left(T^{*}-t\right)\|\omega(t)\|_{\infty}\right)\right)\right) \\
& \left.\leq 6 \mathbf{A}_{d}(t) \pi(t)\left(1+\log ^{+}\left(4\left(T^{*}-t\right)\|\omega(t)\|_{\infty}\right)\right)\right) .
\end{aligned}
$$


For the term $I_{2}$ given by (52), after using the change of variables $z=x+y$, 536 we decompose $I_{2}$ as the sum of two terms $I_{2,1}$ and $I_{2,2}$ defined by

$$
I_{2,1}=c_{d} \int_{B\left(x, \rho_{0}(t)\right) \cap B\left(x, \min \left(\rho(t), \rho_{0}(t)\right)\right)^{c} \cap \mathcal{V}(t)^{c}} \mathbf{D}_{d}(\widehat{z-x}, \omega(z, t), \xi(x, t)) \frac{d z}{|z-x|^{d}}
$$

537 and

$$
I_{2,2}=c_{d} \int_{B\left(x, \rho_{0}(t)\right) \cap B\left(x, \min \left(\rho(t), \rho_{0}(t)\right)\right)^{c} \cap \mathcal{V}(t)} \mathbf{D}_{d}(\widehat{z-x}, \omega(z, t), \xi(x, t)) \frac{d z}{|z-x|^{d}} .
$$

538 Let us estimate the terms $I_{2,1}$ and $I_{2,2}$. For this purpose, we introduce the

539 function $\gamma$ defined for all $t \in\left[0, T^{*}\left[\right.\right.$ by $\gamma(t)=\min \left(\frac{1}{4 A_{0}(t) \Omega(t)\left(T^{*}-t\right)}, \rho_{0}(t)\right)$.

540 From (67), we observe

$$
\begin{aligned}
I_{2,1} & \leq c_{d} \int_{B\left(x, \rho_{0}(t)\right) \cap \mathcal{V}(t)^{c}} \mathbf{D}_{d}(\widehat{z-x}, \xi(z, t), \xi(x, t))^{+}+\frac{|\omega(z, t)|}{|z-x|^{d}} d z \\
& \leq c_{d} \Omega(t) \int_{B\left(x, \rho_{0}(t)\right)} \frac{\mathbf{D}_{d}(\widehat{z-x}, \xi(z, t), \xi(x, t))^{+}}{|z-x|^{d}} d z \\
& =c_{d} \Omega(t) \int_{B(x, \gamma(t))} \frac{\mathbf{D}_{d}(\widehat{z-x}, \xi(z, t), \xi(x, t))^{+}}{|z-x|^{d}} d z \\
& +c_{d} \Omega(t) \int_{B\left(x, \rho_{0}(t)\right) \cap B(x, \gamma(t))^{c}} \frac{\mathbf{D}_{d}(\widehat{z-x}, \xi(z, t), \xi(x, t))^{+}}{|z-x|^{d}} d z .
\end{aligned}
$$

${ }_{541}$ Furthermore, on one hand, by (33) we have for all $z \in B(x, \gamma(t))$,

$$
\mathbf{D}_{d}(\widehat{z-x}, \xi(z, t), \xi(x, t))^{+} \leq A_{0}(t)|z-x|,
$$

${ }_{542}$ and hence, we obtain

$$
\begin{aligned}
\Omega(t) \int_{B(x, \gamma(t))} \frac{\mathbf{D}_{d}(\widehat{z-x}, \xi(z, t), \xi(x, t))^{+}}{|z-x|^{d}} d z & \leq A_{0}(t) \Omega(t) \int_{B(x, \gamma(t))} \frac{d z}{|z-x|^{d-1}} \\
& =|B(0,1)| A_{0}(t) \Omega(t) \gamma(t) \\
& \leq \frac{|B(0,1)|}{4\left(T^{*}-t\right)}
\end{aligned}
$$

${ }_{543}$ where we have used the definition of the function $\gamma$. On the other hand, since 
$\left|\mathbf{D}_{d}(\widehat{z-x}, \xi(z, t), \xi(x, t))\right| \leq 1$, we get

$$
\begin{aligned}
\int_{B\left(x, \rho_{0}(t)\right) \cap B(x, \gamma(t))^{c}} \frac{\mathbf{D}_{d}(\widehat{z-x}, \xi(z, t), \xi(x, t))^{+}}{|z-x|^{d}} d z \\
\leq \int_{B\left(x, \rho_{0}(t)\right) \cap B(x, \gamma(t))^{c}} \frac{d z}{|z-x|^{d}} \\
\quad=|B(0,1)| \int_{\gamma(t)}^{\rho_{0}(t)} \frac{d s}{s} \\
\quad=|B(0,1)| \log \left(\frac{\rho_{0}(t)}{\gamma(t)}\right) \\
\quad=|B(0,1)| \log ^{+}\left(4 \rho_{0}(t) A_{0}(t) \Omega(t)\left(T^{*}-t\right)\right),
\end{aligned}
$$

${ }_{545}$ where we have used again the definition of the function $\gamma$. Owing to (70) and 546 (71), from (69), we deduce

$$
I_{2,1} \leq c_{d}|B(0,1)|\left(\frac{1}{4\left(T^{*}-t\right)}+\Omega(t) \log ^{+}\left(4 \rho_{0}(t) A_{0}(t) \Omega(t)\left(T^{*}-t\right)\right)\right) .
$$

${ }_{547} \quad$ For the term $I_{2,2}$, if $\mathcal{V}(t)=\emptyset$ or if $\rho(t) \geq \rho_{0}(t)$ then from (68) we infer that ${ }_{548} \quad I_{2,2}=0$. Let us assume now that $\mathcal{V}(t) \neq \emptyset$ and $\rho(t)<\rho_{0}(t)$. Then, from (68) 549 we get

$$
\begin{aligned}
I_{2,2} & =c_{d} \int_{\rho(t)}^{\rho_{0}(t)}\left(\int_{\partial B(x, R) \cap \mathcal{V}(t)}|\omega(z, t)| \frac{d \gamma(z)}{|z-x|^{d}}\right) d R \\
& =c_{d} \int_{\rho(t)}^{\rho_{0}(t)} \frac{1}{R^{d}}\left(\int_{\partial B(x, R) \cap \mathcal{V}(t)}|\omega(z, t)| d \gamma(z)\right) d R .
\end{aligned}
$$

By using (60) and an integration by parts we obtain

$$
I_{2,2}=c_{d}\left(\frac{F_{t}\left(\rho_{0}(t)\right)}{\rho_{0}(t)^{d}}-\frac{F_{t}(\rho(t))}{\rho(t)^{d}}+d \int_{\rho(t)}^{\rho_{0}(t)} \frac{F_{t}(R)}{R^{d+1}} d R\right) .
$$

551 Since $\pi(t)=\sup _{0<R \leq \rho_{0}(t)} \frac{F_{t}(R)}{R^{d-1}}$, then from (74) we deduce

$$
\begin{aligned}
I_{2,2} & \leq c_{d}\left(\frac{\pi(t)}{\rho_{0}(t)}+d \pi(t) \int_{\rho(t)}^{\rho_{0}(t)} \frac{d R}{R^{2}}\right) \\
& \leq c_{d}(d+1) \frac{\pi(t)}{\rho(t)},
\end{aligned}
$$

${ }_{552}$ where we have used the fact that $\rho(t)<\rho_{0}(t)$. Therefore whatever the case, we 553 obtain that

$$
I_{2,2} \leq c_{d}(d+1) \frac{\pi(t)}{\rho(t)} .
$$

554 By using (38) the definition of the function $\rho$, from (75) we deduce

$$
I_{2,2} \leq \frac{1}{4\left(T^{*}-t\right)} .
$$




$$
\left|\nabla_{y} \times\left(\frac{(\hat{y} \cdot \xi(x, t))(\xi(x, t) \times \hat{y})}{|y|^{3}}\right)\right| \leq\left|\nabla\left(\frac{\hat{y}}{|y|^{3}}\right)\right|+\frac{\left|\nabla_{y} \times(\xi(x, t) \times \hat{y})\right|}{|y|^{3}} .
$$

${ }_{571}$ We have $\left|\nabla\left(\frac{\hat{y}}{|y|^{3}}\right)\right| \leq \frac{3}{|y|^{4}}$. Furthermore, we have $\nabla_{y} \times(\xi(x, t) \times \hat{y})=\left(\nabla_{y}\right.$. $\left.{ }_{572} \hat{y}\right) \xi(x, t)-\left(\xi(x, t) \cdot \nabla_{y}\right) \hat{y}$ and then we deduce $\left|\nabla_{y} \times(\xi(x, t) \times \hat{y})\right| \leq|\nabla \cdot \hat{y}|+|\nabla \hat{y}| \leq$ ${ }_{573} \frac{3}{|y|}$. After gathering these results, we obtain that for all $y \neq 0$,

$$
\left|\nabla_{y} \times\left(\frac{(\hat{y} \cdot \xi(x, t))(\xi(x, t) \times \hat{y})}{|y|^{3}}\right)\right| \leq \frac{6}{|y|^{4}} .
$$

${ }_{574}$ Therefore, from (78) we obtain

$$
I_{3} \leq 6 c_{3} \int_{B\left(0, \rho_{0}(t)\right)^{c}} \frac{|u(x+y, t)|}{|y|^{4}} d y+c_{3} \int_{\partial B\left(0, \rho_{0}(t)\right)}|u(x+y, t)| \frac{d y}{|y|^{3}} d \gamma(y) .
$$


575 In the case of 2D QG equations for which $d=2$, we get $\mathbf{D}_{2}(\hat{y}, \omega(x+$ $\left.\left.{ }_{576} y, t\right), \xi(x, t)\right)=\left(\hat{y} \cdot \xi(x, t)^{\perp}\right)\left(\omega(x+y, t) \cdot \xi(x, t)^{\perp}\right)$. Since $\omega(x+y, t)=\nabla_{y}^{\perp} u(x+y, t)$, 577 we deduce

$$
\mathbf{D}_{2}(\hat{y}, \omega(x+y, t), \xi(x, t))=\left(\hat{y} \cdot \xi(x, t)^{\perp}\right) \xi(x, t)^{\perp} \cdot \nabla_{y}^{\perp} u(x+y, t) .
$$

578 Then, after using an integration by parts, from (53), we deduce,

$$
\begin{aligned}
I_{3} & =-c_{2} \int_{B\left(0, \rho_{0}(t)\right)^{c}} \nabla_{y}^{\perp} \cdot\left(\frac{\left(\hat{y} \cdot \xi(x, t)^{\perp}\right) \xi(x, t)^{\perp}}{|y|^{2}}\right) u(x+y, t) d y \\
& +c_{2} \int_{\partial B\left(0, \rho_{0}(t)\right)}\left(\frac{\left(\hat{y} \cdot \xi(x, t)^{\perp}\right) \xi(x, t)^{\perp}}{|y|^{2}}\right) \cdot y^{\perp} u(x+y, t) .
\end{aligned}
$$

$579 \quad$ After setting $\psi(y) \equiv \frac{\left(\hat{y} \cdot \xi(x, t)^{\perp}\right)}{|y|^{2}}$ and $\mathbf{V}(y) \equiv \xi(x, t)^{\perp}$, by using the following ${ }_{580}$ vectorial identity $\operatorname{curl}(\psi \mathbf{V})=\nabla^{\perp} \psi \cdot \mathbf{V}+\psi \operatorname{curl} \mathbf{V}$, we obtain after elementary 581 computations, that for all $y \neq 0$,

$$
\begin{aligned}
\left|\nabla_{y}^{\perp}\left(\frac{\left(\hat{y} \cdot \xi(x, t)^{\perp}\right) \xi(x, t)^{\perp}}{|y|^{2}}\right)\right| & \leq\left|\nabla\left(\frac{\hat{y}}{|y|^{2}}\right)\right| \\
\leq & \frac{2}{|y|^{3}} .
\end{aligned}
$$

582 Therefore, from (80) we obtain

$$
I_{3} \leq 2 c_{2} \int_{B\left(0, \rho_{0}(t)\right)^{c}} \frac{|u(x+y, t)|}{|y|^{3}} d y+c_{2} \int_{\partial B\left(0, \rho_{0}(t)\right)} \frac{|u(x+y, t)|}{|y|^{2}} d \gamma(y) .
$$

${ }_{583}$ Therefore, whatever the case considered, 3D Navier-Stokes, 3D Euler or 2D QG ${ }_{584}$ equations, from (79) and (81) we get

$$
I_{3} \leq d(d-1) c_{d} \int_{B\left(0, \rho_{0}(t)\right)^{c}} \frac{|u(x+y, t)|}{|y|^{d+1}} d y+c_{d} \int_{\partial B\left(0, \rho_{0}(t)\right)} \frac{|u(x+y, t)|}{|y|^{d}} d \gamma(y) .
$$

585 Then from (82), we obtain

$$
\begin{aligned}
I_{3} & \leq c_{d}\|u(t)\|_{\infty}\left(d(d-1) \int_{B\left(0, \rho_{0}(t)\right)^{c}} \frac{d y}{|y|^{d+1}}+\int_{\partial B\left(0, \rho_{0}(t)\right)} \frac{d \gamma(y)}{|y|^{d}}\right) \\
& =c_{d}\|u(t)\|_{\infty}\left(d(d-1)|B(0,1)| \int_{\rho_{0}(t)}^{+\infty} \frac{d s}{s^{2}}+\frac{|\partial B(0,1)|}{\rho_{0}(t)}\right) \\
& \leq 9 \frac{\|u(t)\|_{\infty}}{\rho_{0}(t)} .
\end{aligned}
$$

${ }_{586}$ Then, owing to (66), (77) and (83), from (50) we deduce that for any $t \in[0, T]$ ${ }_{587}$ and $x \in \Theta(t)$,

$$
\begin{aligned}
\alpha(x, t) & \left.\leq 6 \mathbf{A}_{d}(t) \pi(t)\left(1+\log ^{+}\left(4\left(T^{*}-t\right)\|\omega(t)\|_{\infty}\right)\right)\right) \\
& +\frac{1}{2\left(T^{*}-t\right)}+\Omega(t) \log ^{+}\left(4 \rho_{0}(t) A_{0}(t) \Omega(t)\left(T^{*}-t\right)\right)+9 \frac{\|u(t)\|_{\infty}}{\rho_{0}(t)} .
\end{aligned}
$$


By using (32), we deduce that for any $t \in[0, T]$ and $x \in \Theta(t)$,

$$
\begin{aligned}
\alpha(x, t) & \left.\leq 6 \mathbf{A}_{d}(t) \pi(t)\left(1+\log ^{+}\left(4\left(T^{*}-t\right)\|\omega(t)\|_{\infty}\right)\right)\right) \\
& +\frac{3}{4\left(T^{*}-t\right)}+\Omega(t) \log ^{+}\left(4 \rho_{0}(t) A_{0}(t) \Omega(t)\left(T^{*}-t\right)\right) .
\end{aligned}
$$

${ }_{589}$ Furthermore, thanks to (35) we get $\left(T^{*}-t\right) \Omega(t) \log ^{+}\left(4 \rho_{0}(t) \mathbf{A}_{0}(t)\right) \leq \frac{1}{8}$ and $590 \quad\left(T^{*}-t\right) \Omega(t)<1$ which implies that $\log ^{+}\left(\left(T^{*}-t\right) \Omega(t)\right)=0$ and hence we obtain ${ }_{591}$ that for all $t \in\left[0, T^{*}[\right.$,

$$
\begin{aligned}
\left(T^{*}-t\right) \Omega(t) \log ^{+} & \left(4 \rho_{0}(t) \mathbf{A}_{0}(t) \Omega(t)\left(T^{*}-t\right)\right) \\
& \leq\left(T^{*}-t\right) \Omega(t)\left(\log ^{+}\left(4 \rho_{0}(t) \mathbf{A}_{0}(t)\right)+\log ^{+}\left(\Omega(t)\left(T^{*}-t\right)\right)\right) \\
& =\left(T^{*}-t\right) \Omega(t) \log ^{+}\left(4 \rho_{0}(t) \mathbf{A}_{0}(t)\right) \\
& \leq \frac{1}{8}
\end{aligned}
$$

${ }_{592}$ Therefore from (85), we deduce that for any $t \in[0, T]$ and $x \in \Theta(t)$,

$$
\alpha(x, t) \leq 6 \mathbf{A}_{d}(t) \pi(t)\left(1+\log ^{+}\left(4\left(T^{*}-t\right)\|\omega(t)\|_{\infty}\right)\right)+\frac{7}{8\left(T^{*}-t\right)} .
$$

593

Then from (49) we deduce

$$
\frac{d}{d t}\|\omega(t)\|_{\infty} \leq\left(6 \mathbf{A}_{d}(t) \pi(t)\left(1+\log ^{+}\left(4\left(T^{*}-t\right)\|\omega(t)\|_{\infty}\right)\right)+\frac{7}{8\left(T^{*}-t\right)}\right)\|\omega(t)\|_{\infty},
$$

594 which is valid for all $t \in[0, T]$ and $T<T^{*}$ and then inequality (87) is valid for ${ }_{595}$ all $t \in\left[0, T^{*}\right.$. Let $t_{0} \in\left[0, T^{*}[\right.$ such that

$$
4\left(T^{*}-t_{0}\right) \leq 1 .
$$

596 Then we get that for all $t \in\left[t_{0}, T^{*}\left[, 4\left(T^{*}-t\right) \leq 1\right.\right.$ and hence

$$
\begin{aligned}
\left.\log ^{+}\left(4\left(T^{*}-t\right)\|\omega(t)\|_{\infty}\right)\right) & \leq \log ^{+}\left(4\left(T^{*}-t\right)\right)+\log ^{+}\|\omega(t)\|_{\infty} \\
& =\log ^{+}\|\omega(t)\|_{\infty} .
\end{aligned}
$$

597 Owing to (89), from (87) we deduce that for all $t \in\left[t_{0}, T^{*}[\right.$

$$
\frac{d}{d t}\|\omega(t)\|_{\infty} \leq\left(6 \mathbf{A}_{d}(t) \pi(t)\left(1+\log ^{+}\|\omega(t)\|_{\infty}\right)+\frac{7}{8\left(T^{*}-t\right)}\right)\|\omega(t)\|_{\infty} .
$$

${ }_{598}$ Thanks to Grönwall inequality, from (90) we deduce that for all $t \in\left[t_{0}, T^{*}[\right.$,

$$
\begin{aligned}
\|\omega(t)\|_{\infty} & \leq\left\|\omega\left(t_{0}\right)\right\|_{\infty} e^{\int_{t_{0}}^{t}\left(6 \mathbf{A}_{d}(\tau) \pi(\tau)\left(1+\log ^{+}\|\omega(\tau)\|_{\infty}\right)+\frac{7}{8\left(T^{*}-\tau\right)}\right) d \tau} \\
& =\left(\frac{T^{*}-t_{0}}{T^{*}-t}\right)^{\frac{7}{8}}\left\|\omega\left(t_{0}\right)\right\|_{\infty} e^{\int_{t_{0}}^{t} 6 \mathbf{A}_{d}(\tau) \pi(\tau)\left(1+\log ^{+}\|\omega(\tau)\|_{\infty}\right) d \tau} .
\end{aligned}
$$


Since the function $z \mapsto \log ^{+}(z)$ is non-decreasing on $] 0,+\infty[$, then after applying the function $1+\log ^{+}$to the inequality (91) and using the fact that $\log ^{+}(a b) \leq$ $\log ^{+} a+\log ^{+} b$, we thus obtain that for all $t \in\left[t_{0}, T^{*}[\right.$

$$
\begin{aligned}
1+\log ^{+}\|\omega(t)\|_{\infty} & \leq 1+\log \left(\left(\frac{T^{*}-t_{0}}{T^{*}-t}\right)^{\frac{7}{8}}\right)+\log ^{+}\left\|\omega\left(t_{0}\right)\right\|_{\infty} \\
& +6 \int_{t_{0}}^{t} \mathbf{A}_{d}(\tau) \pi(\tau)\left(1+\log ^{+}\|\omega(\tau)\|_{\infty}\right) d \tau
\end{aligned}
$$

${ }_{602}$ Since the function $t \mapsto \log \left(\left(\frac{T^{*}-t_{0}}{T^{*}-t}\right)^{\frac{7}{8}}\right)$ is increasing over $\left[t_{0}, T^{*}[\right.$ then thanks to Gronwall Lemma, by (92) we deduce that for all $t \in\left[t_{0}, T^{*}[\right.$,

$$
1+\log ^{+}\|\omega(t)\|_{\infty} \leq\left(\log \left(\left(\frac{T^{*}-t_{0}}{T^{*}-t}\right)^{\frac{7}{8}}\right)+1+\log ^{+}\left\|\omega\left(t_{0}\right)\right\|_{\infty}\right) e^{6 \int_{t_{0}}^{t} \mathbf{A}_{d}(\tau) \pi(\tau) d \tau} .
$$

$$
\begin{aligned}
\|\omega(t)\|_{\infty} & \leq \exp \left(\left(\log \left(\left(\frac{T^{*}-t_{0}}{T^{*}-t}\right)^{\frac{7}{8}}\right)+1+\log ^{+}\left\|\omega\left(t_{0}\right)\right\|_{\infty}\right) e^{6 \int_{t_{0}}^{t} \mathbf{A}_{d}(\tau) \pi(\tau) d \tau}\right) \\
& =\exp \left(\left(1+\log ^{+}\left\|\omega\left(t_{0}\right)\right\|_{\infty}\right) e^{6 \int_{t_{0}}^{t} \mathbf{A}_{d}(\tau) \pi(\tau) d \tau}\right)\left(\frac{T^{*}-t_{0}}{T^{*}-t}\right)^{\frac{7}{8} e^{6 \int_{t_{0}}^{t} \mathbf{A}_{d}(\tau) \pi(\tau) d \tau}} .
\end{aligned}
$$

${ }_{606}$ Let us assume that there exists $t_{1} \in\left[0, T^{*}\left[\right.\right.$ such that $\int_{t_{1}}^{T^{*}} \mathbf{A}_{d}(\tau) \pi(\tau) d \tau<$ ${ }_{607}+\infty$. Then, in addition of $(88)$, we choose $t_{0} \in\left[t_{1}, T^{*}\left[\right.\right.$ such that $M_{t_{0}} \stackrel{\text { def }}{=}$ ${ }_{608} \int_{t_{0}}^{T^{*}} \mathbf{A}_{d}(\tau) \pi(\tau) d \tau<\frac{1}{6} \log \left(\frac{8}{7}\right)$. We thus get for all $t \in\left[t_{0}, T^{*}[\right.$,

$$
\frac{7}{8} e^{6 \int_{t_{0}}^{t} \mathbf{A}_{d}(\tau) \pi(\tau) d \tau} \leq \frac{7}{8} e^{6 M_{t_{0}}}<1
$$

${ }_{609}$ Therefore with $\eta_{t_{0}} \stackrel{\text { def }}{=} \frac{7}{8} e^{6 M_{t_{0}}}$, from (94) we deduce that for all $t \in\left[t_{0}, T^{*}[\right.$

$$
\|\omega(t)\|_{\infty} \leq \exp \left(\left(1+\log ^{+}\left\|\omega\left(t_{0}\right)\right\|_{\infty}\right) e^{6 M_{t_{0}}}\right)\left(\frac{T^{*}-t_{0}}{T^{*}-t}\right)^{\eta_{t_{0}}}
$$

${ }_{610}$ Since $\eta_{t_{0}}<1$, from (95) we thus deduce that $\int_{t_{0}}^{T^{*}}\|\omega(t)\|_{\infty} d t<+\infty$. Since $u \in$ ${ }_{611} C\left(\left[0, T^{*}\left[; H_{\sigma}^{r}\left(\mathbb{R}^{d}\right)\right)\right.\right.$ and thanks to the Sobolev embedding $H^{r}\left(\mathbb{R}^{d}\right) \hookrightarrow B C^{3}\left(\mathbb{R}^{d}\right)$ ${ }_{612}$ due to $r>\frac{d}{2}+3$, we infer that $\omega \in C\left(\left[0, T^{*}\left[; B C^{2}\left(\mathbb{R}^{d}\right)\right)\right.\right.$ which implies that ${ }_{613} \int_{0}^{t_{0}}\|\omega(t)\|_{\infty} d t<+\infty$. Therefore we deduce that $\int_{0}^{T^{*}}\|\omega(t)\|_{\infty} d t<+\infty$. ${ }_{614}$ If $u$ blows up at the finite time $T^{*}$ then thanks to $(20)$ and $(23)$ we have 
${ }_{615} \int_{0}^{T^{*}}\|\omega(t)\|_{\infty} d t=+\infty$ which leads to a contradiction. Then, we deduce that $u$ ${ }_{616}$ cannot blow up at the time $T^{*}$ which concludes the first part of proof.

${ }_{617}$ Thanks to (44), we have already $\nabla|\omega|(x, t)=0$. Since $\nabla \cdot \omega=0$ and $\omega=|\omega| \xi$, ${ }_{618}$ then we get

$$
0=\nabla \cdot \omega=|\omega| \nabla \cdot \xi+\xi \cdot \nabla|\omega| .
$$

However, for all $x \in \Theta(t),|\omega(x, t)|=\|\omega(t)\|_{\infty}>0$ and from (44), we have $\nabla|\omega|(x, t)=0$. Therefore, from (96), we deduce that for all $t \in[0, T]$ and $x \in \Theta(t)$,

$$
\nabla \cdot \xi(x, t)=0
$$

which completes the proof.

\section{No blow up in finite time for numerical experiments}

In this section, we show the non-blowup in finite time of the solutions of the 3D Euler equations in the numerical experiments considered these last years.

First, we emphasize that the singularity discovered in Luo and Hou (2014b) which lies right on the boundary is not relevant in the case of the whole domain $\mathbb{R}^{3}$. Indeed recently, the authors found a convincing numerical evidence for a singular solution to the Euler equations in a fluid with periodic boundary condition along the axial direction and no-flow boundary condition on the solid wall Luo and Hou (2014b) (see also Luo and Hou (2014a)), for which the point of the potential singularity, which is also the point of the maximum vorticity, is always located at the solid boundary. However thanks to Theorem 5.1, we deduce that such singularity can not exist in the whole domain $\mathbb{R}^{3}$. Indeed, in the whole domain of $\mathbb{R}^{3}$ at any point of the maximum vorticity, $q_{0} \in \mathbb{R}^{3}$, thanks to Theorem 5.1 we get $\nabla|\omega|\left(q_{0}, t\right)=0$ for any time $t$ before the alleged time of singularity $T^{*}$, then this result combined with the fact that the vorticity $\omega$ is a divergence-free vector field, yields to get $\nabla \cdot \xi\left(q_{0}, t\right)=0$ in Theorem 5.1. However in Luo and Hou (2014b), the presence of a solid boundary and the fact that $q_{0}$ the point of the maximum vorticity is always located on the solid boundary, prevent to get $\nabla|\omega|\left(q_{0}, t\right)=0$ and this allows to get $\nabla \cdot \xi\left(q_{0}, t\right) \sim\left(T^{*}-t\right)^{-2.9165} \neq 0$ as it is observed in their numerical test. This latter is the main element used to invalidate the Deng-Hou-Yu non-blowup criterion Deng et al. (2005, 2006a).

There have been many computational attempts to find finite-time singularities of the 3D Euler and Navier-Stokes equations: see, e.g Melander and Hussain (1989); Kerr and Hussain (1989); Pumir and Siggia (1990); Kerr (1993); Grauer et al. (1998); Hou and Li (2006); Kerr (2005). One example that has been studied extensively in these numerical investigations is the interaction of two perturbed antiparallel vortex tubes. All the subsequent calculations assumed an anti-parallel geometry, for which there are two symmetry planes. One in $y-z$ is between the vortices and was called the 'dividing plane'. The other in $x-z$ is at the position of maximum perturbation and was called the 'symmetry plane'. The difficulty faced in each computational attempts cited was to find a better initial condition 
within this geometry (see Kerr (2006)). From these computational attempts, a numerical controversy takes place around the question to know whether or not there is finite-time blow-up of the solutions of Euler equations (see Kerr (2006)).

In this section, we propose an answer to this controversy by using our Theorem 5.1. By using the anisotropic structure of regions of high vorticity described in Kerr $(2005,1998)$, we show straightforward thanks to our Theorem 5.1 that the solutions of Euler equations cannot blow up in finite time in these numerical experiments Kerr $(2005,1998,1997)$.

For this purpose, we give a first bound of the function $\pi$ defined by (37) in the following Lemma. The bound given in Lemma 6.1 of the function $\pi$ is not a sharp bound but obtained without assumptions.

Lemma 6.1. Let $d \in\{2,3\}, u_{0} \in H_{\sigma}^{r}\left(\mathbb{R}^{d}\right)$ with $r>\frac{d}{2}+3$. Let $T^{*}>0$ be such that there exists a unique strong solution $u$ to the $3 D$ Navier-Stokes, $3 D$ Euler equations (9)-(10) or $2 D$ QG equations (11)-(12) in the class

$$
u \in C\left(\left[0, T^{*}\left[; H_{\sigma}^{r}\left(\mathbb{R}^{d}\right)\right) \cap C^{1}\left(\left[0, T^{*}\left[; H^{r-2}\left(\mathbb{R}^{d}\right)\right) .\right.\right.\right.\right.
$$

Under the definitions (32)-(37) in the Theorem 5.1, we have the following estimate: for all $t \in\left[0, T^{*}[\right.$

$$
\pi(t) \leq 3\|\omega(t)\|_{\infty} \sup _{x \in \Theta(t)}\left|\mathcal{V}(t) \cap B\left(x, \rho_{0}(t)\right)\right|^{\frac{1}{d}} .
$$

${ }_{61}$ Proof. For any $t \in\left[0, T^{*}\left[, x \in \Theta(t)\right.\right.$ and $0<R \leq \rho_{0}(t)$, we get

$$
\frac{1}{R^{d-1}} \int_{B(x, R) \cap \mathcal{V}(t)}|\omega(z, t)| d z \leq \frac{1}{R^{d-1}}\|\omega(t)\|_{\infty}|\mathcal{V}(t) \cap B(x, R)| .
$$

672 Furthermore, we have

$$
\begin{aligned}
|\mathcal{V}(t) \cap B(x, R)| & =|\mathcal{V}(t) \cap B(x, R)|^{\frac{1}{d}}|\mathcal{V}(t) \cap B(x, R)|^{\frac{d-1}{d}} \\
& \leq|\mathcal{V}(t) \cap B(x, R)|^{\frac{1}{d}}|B(x, R)|^{\frac{d-1}{d}} \\
& =|B(0,1)|^{\frac{d-1}{d}}|\mathcal{V}(t) \cap B(x, R)|^{\frac{1}{d}} R^{d-1} \\
& \leq 3|\mathcal{V}(t) \cap B(x, R)|^{\frac{1}{d}} R^{d-1}
\end{aligned}
$$

${ }_{673}$ where we have used the fact that $|B(0,1)|=\left(\frac{4 \pi}{3}\right)^{\frac{2}{3}}$ if $d=3$ or $|B(0,1)|=\pi^{\frac{1}{2}}$ 674 if $d=2$.

675 Then, we deduce for that any $t \in\left[0, T^{*}\left[, x \in \Theta(t)\right.\right.$ and $0<R \leq \rho_{0}(t)$,

$$
\frac{1}{R^{d-1}} \int_{B(x, R) \cap \mathcal{V}(t)}|\omega(z, t)| d z \leq 3\|\omega(t)\|_{\infty}|\mathcal{V}(t) \cap B(x, R)|^{\frac{1}{d}} .
$$

Owing to (98), we thus conclude the proof. 
Now, we can show straightforward thanks to Theorem 5.1 and Lemma 6.1 that the solutions of Euler equations cannot blow up in finite time in the numerical experiments Kerr $(2005,1998,1997)$.

For this purpose, we recall that in the numerical experiments Kerr $(2005,1998)$, the author show that the blow-up rates at some time $T^{*}$ the alleged time of singularity, to be considered for $\|\omega(t)\|_{\infty},\|u(t)\|_{\infty}$ and $\|\nabla \xi(t)\|_{\infty}$ in $\operatorname{Kerr}(2005$, 1998) are

$$
\|\omega(t)\|_{\infty} \sim\left(T^{*}-t\right)^{-1},\|u(t)\|_{\infty} \sim\left(T^{*}-t\right)^{-\frac{1}{2}} \text { and }\|\nabla \xi(t)\|_{\infty} \sim\left(T^{*}-t\right)^{-\frac{1}{2}},
$$

for time $t \in\left[t_{0}, T^{*}\left[\right.\right.$ with $t_{0} \in\left[0, T^{*}\left[\right.\right.$ sufficiently close to $T^{*}$. Moreover for time $t \in\left[t_{0}, T^{*}\left[\right.\right.$ with $t_{0}$ sufficiently close to $T^{*}$, the author showed that the support of the maximum vorticity

$$
\mathcal{E}(t)=\left\{x \in \mathbb{R}^{3},|\omega(x, t)| \sim\|\omega(t)\|_{\infty}\right\}
$$

is characterized by two length scales $\left(T^{*}-t\right)$ and $\left(T^{*}-t\right)^{\frac{1}{2}}$ and its volume is bounded by

$$
|\mathcal{E}(t)| \lesssim\left(T^{*}-t\right)^{2}
$$

Thanks to the blow-up rates (99), from (32) we get that for all $t \in\left[t_{0}, T^{*}[\right.$

$$
\rho_{0}(t) \sim\left(T^{*}-t\right)^{\frac{1}{2}} .
$$

From (33), thanks again to (99) we have that for all $t \in\left[t_{0}, T^{*}[\right.$

$$
\begin{aligned}
\mathbf{A}_{0}(t) & \leq \sup _{x \in \Theta(t)}\|\nabla \xi(t)\|_{L^{\infty}\left(B\left(x, \rho_{0}(t)\right)\right)} \\
& \lesssim\left(T^{*}-t\right)^{-\frac{1}{2}} .
\end{aligned}
$$

Then we deduce that for all $t \in\left[t_{0}, T^{*}[\right.$

$$
\mathbf{A}_{0}(t) \rho_{0}(t) \lesssim 1 .
$$

Therefore thanks to (101), from the definition (35) of the function $\Omega$, we deduce for all $t \in\left[t_{0}, T^{*}[\right.$

$$
\Omega(t) \gtrsim\left(T^{*}-t\right)^{-1} .
$$

694 Owing to (102) and since for all $t \in\left[t_{0}, T^{*}\left[,\|\omega(t)\|_{\infty} \sim\left(T^{*}-t\right)^{-1}\right.\right.$ thanks to ${ }_{695}$ (99), for the set $\mathcal{V}(t)$ defined by (36) we deduce that for all $t \in\left[t_{0}, T^{*}[\right.$

$$
\mathcal{V}(t)=\left\{x \in \mathbb{R}^{3},|\omega(x, t)| \sim\|\omega(t)\|_{\infty}\right\} .
$$

996 Then thanks to (100) we get that for all $t \in\left[t_{0}, T^{*}[\right.$

$$
|\mathcal{V}(t)| \lesssim\left(T^{*}-t\right)^{2} .
$$

Thanks to Lemma 6.1, inequality (103) and the fact that $\|\omega(t)\|_{\infty} \sim\left(T^{*}-t\right)^{-1}$ thanks to (99), for the function $\pi$ defined by (37), we get that for all $t \in\left[t_{0}, T^{*}[\right.$

$$
\pi(t) \lesssim\left(T^{*}-t\right)^{-\frac{1}{3}} .
$$


Furthermore, thanks to (99), for the function $\mathbf{A}_{3}$ defined by (39) for $d=3$, we get that for all $t \in\left[t_{0}, T^{*}[\right.$

$$
\mathbf{A}_{3}(t) \leq\|\nabla \xi(t)\|_{\infty} \lesssim\left(T^{*}-t\right)^{-\frac{1}{2}} .
$$

Owing to (104) and (105) we deduce that for all $t \in\left[t_{0}, T^{*}[\right.$

$$
\mathbf{A}_{3}(t) \pi(t) \lesssim\left(T^{*}-t\right)^{-\frac{5}{6}}
$$

Then, we deduce

$$
\int_{t_{0}}^{T^{*}} \mathbf{A}_{3}(t) \pi(t) \lesssim\left(T^{*}-t_{0}\right)^{\frac{1}{6}}<+\infty .
$$

Therefore, thanks to (107) and Theorem 5.1, we deduce that the solutions of the Euler equations considered for the numerical experiments Kerr $(2005,1998)$ cannot blow-up in finite time at the alleged time of singularity $T^{*}$. If one considers the plausible scenario of blow up proposed in Brenner et al. (2016), one observed that we get also the blow-up rates (99) and the estimate (100) (see (Hormoz and Brenner, 2012, section 4)), hence the potential mechanism proposed for the blow-up in finite time of solutions of Euler equations in Brenner et al. (2016) cannot in fact lead to the blow-up in finite time of the solutions of Euler equations.

\section{Toward the non blowup in finite time of the solutions}

In this section, under mild assumptions deriving from the structure of the regions of high vorticity, we obtain the non-blowup in finite time at some time $T^{*}$ of the solutions of $2 \mathrm{D}$ QG, 3D Euler and 3D Navier-Stokes equations in the case where

$$
\|\nabla \xi(t)\|_{\infty} \sim\left(T^{*}-t\right)^{-\gamma_{\xi}}, 0 \leq \gamma_{\xi}<1 .
$$

In the previous section, we have outlined that the estimate obtained in Lemma 6.1 for the function $\pi$ defined by (37) is not sharp, then in the subsection 7.2 we propose a better estimate for the function $\pi$ and go further in the non blow-up criteria. However, before to deal with new non blow-up criteria in the subsection 7.2 we need to introduce in the subsection 7.1 the Lagrangian flow map $X$ and the definitions of vortex lines and vortex tubes in order to justify the assumption (121) used in Proposition 7.1 and for their use in Lemmata 7.2, 7.3, 7.4 and in the Proposition 7.2.

\subsection{Lagrangian flow map, vortex lines and vortex tubes}

Let $d \in\{2,3\}, u_{0} \in H_{\sigma}^{r}\left(\mathbb{R}^{d}\right)$ with $r>\frac{d}{2}+2$. Let $T^{*}>0$ be such that there exists a unique strong solution $u$ to the $3 \mathrm{D}$ Euler equations (9)-(10) or 2D QG equations (11)-(12) in the class

$$
u \in C\left(\left[0, T^{*}\left[; H_{\sigma}^{r}\left(\mathbb{R}^{d}\right)\right) \cap C^{1}\left(\left[0, T^{*}\left[; H^{r-1}\left(\mathbb{R}^{d}\right)\right) .\right.\right.\right.\right.
$$


Solutions in this class exist thanks to section 3. We set $\boldsymbol{v}=u$ in the case of 3D Euler equations and $v=R^{\perp} u$ in the case of $2 \mathrm{D}$ QG equation.

Owing to $u \in C\left(\left[0, T^{*}\left[, H_{\sigma}^{r}\left(\mathbb{R}^{d}\right)\right) \cap C^{1}\left(\left[0, T^{*}\left[, H^{r-1}\left(\mathbb{R}^{d}\right)\right)\right.\right.\right.\right.$ with $r>\frac{d}{2}+2$ and thanks to the $L^{2}$-boundedness of the Riesz transforms, we infer that $\boldsymbol{v} \in C\left(\left[0, T^{*}\left[, H^{r}\left(\mathbb{R}^{d}\right)\right) \cap C^{1}\left(\left[0, T^{*}\left[, H^{r-1}\left(\mathbb{R}^{d}\right)\right)\right.\right.\right.\right.$ with $r>\frac{d}{2}+2$. Then by using the Sobolev embedding, $H^{m}\left(\mathbb{R}^{d}\right) \hookrightarrow B C^{n}\left(\mathbb{R}^{d}\right)$ with $n=\left[m-\frac{d}{2}\right]$ and $m>\frac{d}{2}$, we deduce that for any $0<T<T^{*}$,

$$
u \in B C^{1}\left(\mathbb{R}^{d} \times[0, T]\right), \nabla u \in C\left(\left[0, T^{*}\left[; B C^{1}\left(\mathbb{R}^{d}\right)\right)\right.\right.
$$

and

$$
\boldsymbol{v} \in B C^{1}\left(\mathbb{R}^{d} \times[0, T]\right), \nabla \boldsymbol{v} \in C\left(\left[0, T^{*}\left[; B C^{1}\left(\mathbb{R}^{d}\right)\right) .\right.\right.
$$

In Proposition 7.1, in the case of 3D Euler equations and 2D QG equations, we give an estimate of the function $\pi$ defined by (37). For this purpose, we need to give the definition of a vortex line and recall some results about the Lagrangian flow map.

We thus introduce the flow map $X(\alpha, \tau, t)$ the particle path that passes by $\alpha \in \mathbb{R}^{d}$ at time $\tau \in\left[0, T^{*}\left[\right.\right.$. That is for $\tau \in\left[0, T^{*}[\right.$ fixed, $X(\alpha, \tau, t)$ solves on $\left[0, T^{*}[\right.$ (see chapter 4 in Majda and Bertozzi (2002) for more details on the flow map $X$ )

$$
\begin{aligned}
\frac{\partial X(\alpha, \tau, t)}{\partial t} & =\boldsymbol{v}(X(\alpha, \tau, t), t), \\
X(\alpha, \tau, \tau) & =\alpha \in \mathbb{R}^{d},
\end{aligned}
$$

Thanks to Cauchy-Lipschitz Theorem (see Theorems 2.2 and 2.13 in Teschl (2012)), for any $\alpha \in \mathbb{R}^{d}$ and $\tau \in\left[0, T^{*}[\right.$ thanks to (110) we get that there exists an unique solution $X(\alpha, \tau, \cdot) \in C^{1}\left(\left[0, T^{*}[)\right.\right.$ to equation (111). For all $t \in\left[0, T^{*}\left[\right.\right.$ and $\tau \in\left[0, T^{*}[\right.$, the map $X(\cdot, \tau, t)$ defined by equation (111) is a volume preserving $C^{1}$-diffeomorphism from $\mathbb{R}^{d}$ on itself. Indeed thanks to (110) and the Theorems 2.2, 2.10 and 2.13 in Teschl (2012), we deduce that for any $t \in\left[0, T^{*}\left[\right.\right.$ and $\tau \in\left[0, T^{*}\left[, X(\cdot, \tau, t)\right.\right.$ is a $C^{1}$-diffeomorphism from $\mathbb{R}^{d}$ on itself with inverse $X(\cdot, t, \tau)$, we notice also $X \in C^{1}\left(\mathbb{R}^{d} \times\left[0, T^{*}\left[\times\left[0, T^{*}[)\right.\right.\right.\right.$. Moreover for any $t \in\left[0, T^{*}\left[\right.\right.$ and $\tau \in\left[0, T^{*}[, X(\cdot, \tau, t)\right.$ is a volume preserving mapping thanks to Proposition 1.4 in Majda and Bertozzi (2002), for which we get

$$
\operatorname{det}\left(\nabla_{\alpha} X(\alpha, \tau, t)\right)=1 .
$$

Furthermore, we have for all $\tau \in\left[0, T^{*}\left[, t \in\left[0, T^{*}\left[\right.\right.\right.\right.$ and $\alpha \in \mathbb{R}^{d}$ (see Proposition 1.8 in Majda and Bertozzi (2002) or Proposition page 24 in Chorin and Marsden (1993) for Euler equations and see Deng et al. (2006b) for 2D QG equation),

$$
\omega(X(\alpha, \tau, t), t)=\nabla_{\alpha} X(\alpha, \tau, t) \omega(\alpha, \tau) .
$$

Recall that a vortex line in a fluid is an arc on an integral curve of the vorticity $\omega(x, t)$ for fixed $t$, and a vortex tube is a tubular neighborhood in $\mathbb{R}^{d}, d \in\{2$, 
arising as a union of vortex lines. In what follows, we give a parametrization of vortex lines and vortex tubes.

We set $\mathcal{O} \stackrel{\text { def }}{=}\left\{\left(x, \tau^{\prime}\right) \in \mathbb{R}^{d} \times\left[0, T^{*}\left[;\left|\omega\left(x, \tau^{\prime}\right)\right|>0\right\}\right.\right.$ and for any $t \in\left[0, T^{*}[\right.$, $\mathcal{O}(t) \stackrel{\text { def }}{=}\left\{x \in \mathbb{R}^{d} ;|\omega(x, t)|>0\right\}$. From $(25)$, we get that for any $t \in\left[0, T^{*}[, \mathcal{O}(t)\right.$ is nonempty. Thanks to (109), we get that $\omega$ is continuous on $\mathbb{R}^{d} \times\left[0, T^{*}[\right.$ and then we deduce that $\mathcal{O}$ is an open subset of $\mathbb{R}^{d} \times\left[0, T^{*}[\right.$ and also that for all $t \in\left[0, T^{*}\left[, \mathcal{O}(t)\right.\right.$ is an open subset of $\mathbb{R}^{d}$. Notice thanks again to (109) that $\xi$ and $\nabla \xi$ are continuous on $\mathcal{O}$. Then, we get that for all $t \in\left[0, T^{*}\left[, \xi(\cdot, t) \in C^{1}(\mathcal{O}(t))\right.\right.$. Then, for all $t \in\left[0, T^{*}\left[\right.\right.$ and $\alpha \in \mathcal{O}(t)$, we denote by $\mathbf{x}_{t}(\alpha, \cdot): \mathfrak{J}_{\alpha, t} \longmapsto \mathbb{R}^{d}$ the vortex line that passes through $\alpha$ at the time $t$ and defined by the ordinary differential equation:

$$
\begin{aligned}
\frac{\partial \mathbf{x}_{t}(\alpha, s)}{\partial s} & =\xi\left(\mathbf{x}_{t}(\alpha, s), t\right), \\
\mathbf{x}_{t}(\alpha, 0) & =\alpha .
\end{aligned}
$$

The set $\mathfrak{J}_{\alpha, t} \subset \mathbb{R}$ not reduced to $\{0\}$ denotes the maximal interval of existence of the unique solution $\mathbf{x}_{t}(\alpha, \cdot)$ of (114), this is ensured thanks to Cauchy-Lipschitz Theorem (see e.g Theorems 2.2 and 2.13 in Teschl (2012)). For any $t \in\left[0, T^{*}[\right.$, we introduce $U_{t}=\left\{(\alpha, s) \in \mathcal{O}(t) \times \mathbb{R} ; \mathbf{x}_{t}(\alpha, s) \in \mathcal{O}(t)\right\}$ the set of definition of the function $\mathbf{x}_{t}$. For any $t \in\left[0, T^{*}\left[\operatorname{since} \xi(\cdot, t) \in C^{1}(\mathcal{O}(t))\right.\right.$, then from Theorem 2.9 in Teschl (2012), we get that $\mathbf{x}_{t}$ is continuous on $U_{t}$. We notice that $U_{t}=\mathbf{x}_{t}^{-1}(\mathcal{O}(t))$ and hence we obtain that $U_{t}$ is an open subset of $\mathcal{O}(t) \times \mathbb{R}$. From Theorem 2.10 in Teschl (2012), we obtain that

$$
\mathbf{x}_{t} \in C^{1}\left(U_{t}\right) .
$$

Any vortex tube $\mathfrak{T}$ at a time $t \in\left[0, T^{*}\left[\right.\right.$ can be defined as $\mathfrak{T} \stackrel{\text { def }}{=}\left\{\mathbf{x}_{t}(\alpha, s) ; \alpha \in\right.$ $\left.\mathcal{A}_{0}, s \in I_{\alpha, t} \subset \mathfrak{J}_{\alpha, t}\right\}$ where $\mathcal{A}_{0}$ is a bounded smooth surface (resp. curve) of $\mathbb{R}^{3}$ (resp. of $\mathbb{R}^{2}$ ) and for each $\alpha \in \mathcal{A}_{0}, I_{\alpha, t}$ is an interval of $\mathbb{R}$ containing 0 .

\subsection{Anisotropic structure for the improvement of non blow-up criteria}

In this subsection, in Proposition 7.1 we propose to show that the function $\pi(t)$ defined by (37) involved Theorem 5.1 is bounded by $C\left(1+\log ^{+}\left(\frac{\|\omega(t)\|_{\infty}}{\Omega(t)}\right)\right)$ by using assumptions related to the anisotropic scaling in the collapse of regions of high vorticity containing the positions of the maximum vorticity. In Proposition 7.2, in the case of the Euler and 2D QG equations, we improve logarithmically the result obtained in Proposition 7.1 by showing that the function $\pi$ is bounded.

These results come from the special feature of the structure of regions of high vorticity surrounding the peak of vorticity $\left\{y \in \mathbb{R}^{d} ;|\omega(y, t)| \gtrsim\|\omega(t)\|_{\infty}\right\}$ observed in the numerical experiments Kerr (1998, 2005); Agafontsev et al. (2015, 2017) and from analytical models (Agafontsev et al., 2017, section 3),

(Majda and Bertozzi, 2002, sections 1.4 and 1.5), namely they are pancakelike structure characterized by two length scales whose one of it is bounded by $O\left(\frac{1}{\|\omega(t)\|_{\infty}}\right)$ and plays the role of the thickness of the pancake-like structure. 
This suggests that for any $t \in\left[t_{0}, T^{*}\left[\right.\right.$ with $t_{0} \in\left[0, T^{*}[, x \in \Theta(t), \lambda \geq \Omega(t)\right.$ the set $\left\{y \in \mathbb{R}^{d} ;|\omega(y, t)| \geq \lambda\right\}$ may be characterized by three length scales whose one of them is of order $\frac{1}{\lambda}$, where $\Omega$ and $\Theta$ are respectively defined by (35) and (34). Since for any $0<R \leq \rho_{0}(t)$ the set $\mathcal{V}_{\lambda, R}(t) \stackrel{\text { def }}{=}\{y \in B(x, R) ;|\omega(y, t)| \geq$ $\lambda\} \subset B(x, R)$, we thus expect that the set $\mathcal{V}_{\lambda, R}(t)$ may have two of its length scales of order $R$ and the third one of order $\frac{1}{\lambda}$. Then we expect that for for any $t \in\left[t_{0}, T^{*}\left[, x \in \Theta(t), \lambda \geq \Omega(t)\right.\right.$ and $0<R \leq \rho_{0}(t)$,

$$
\left|\mathcal{V}_{\lambda, R}(t)\right| \lesssim t_{0} \frac{R^{d-1}}{\lambda}
$$

In Lemma 7.1 we give an argument in favour of the assumption (116) in the case of 3D Euler equations and 2D QG equation by using their Lagrangian structure. In Lemma 7.1, the property (P1) expresses the fact that we expect the length of any segment of a vortex line included in the structure $\mathcal{V}_{\lambda, R}(t)$ is bounded by $O(R)$, since $\mathcal{V}_{\lambda, R}(t) \subset B(x, R)$. Furthermore property (P2) expresses the pancake structure of regions of high vorticity observed in numerical experiments. Indeed for the case of 3D Euler equations, if one assumes that the set $\mathcal{V}_{\lambda, R}^{0}(t) \stackrel{\text { def }}{=} X^{-1}\left(\mathcal{V}_{\lambda, R}(t), t_{0}, t\right)$ is characterized by three length scales $\ell_{1}^{0}, \ell_{2}^{0}, \ell_{3}^{0}$ associated to three main directions orthogonal between them pairwise, then we should have for one of these length scales $\ell_{1}^{0} \lesssim \ell_{\lambda, R}^{0}(t)$ or $\ell_{2}^{0} \lesssim \ell_{\lambda, R}^{0}(t)$ or $\ell_{3}^{0} \lesssim \ell_{\lambda, R}^{0}(t)$ (117). Let us say that $\ell_{1}^{0} \lesssim \ell_{\lambda, R}^{0}(t)$. Assuming that during the time between $t_{0}$ and $\left.t \in\right] t_{0}, T^{*}\left[\right.$ the set $\mathcal{V}_{\lambda, R}^{0}(t)$ becomes a pancake-shaped structure, we thus expect that $\ell_{2}^{0} \lesssim R$ or $\ell_{3}^{0} \lesssim R$ since $\mathcal{V}_{\lambda, R}(t) \subset B(x, R)$. Let us say that $\ell_{2}^{0} \lesssim R$. For the last length scale $\ell_{3}^{0}$, we just expect that $\ell_{3}^{0}=O(1)$.

In the case of $3 \mathrm{D}$ Euler equations, we thus expect that $\left|\mathcal{V}_{\lambda, R}^{0}\left(t_{0}\right)\right| \lesssim \ell_{\lambda, R}^{0}(t) R$.

In the case of 2D QG equations, we will have only the two length scales $\ell_{1}^{0}$ and $\ell_{3}^{0}$ and then we expect that $\left|\mathcal{V}_{\lambda, R}^{0}\left(t_{0}\right)\right| \lesssim \ell_{\lambda, R}^{0}(t)$. Then Lemma 7.1 gives an explanation of assumption (116).

Lemma 7.1. Under the definitions (31)-(35) in the Theorem 5.1, we assume that there exists $t_{0} \in\left[0, T^{*}\left[\right.\right.$ such that for any $t \in\left[t_{0}, T^{*}\left[, 0<R \leq \rho_{0}(t)\right.\right.$ and $\lambda \geq \Omega(t)$ the sets $\mathcal{V}_{\lambda, R}(t) \stackrel{\text { def }}{=}\{y \in B(x, R) ;|\omega(y, t)| \geq \lambda\}$ and $\mathcal{V}_{\lambda, R}^{0}(t) \stackrel{\text { def }}{=}$ $X^{-1}\left(\mathcal{V}_{\lambda, R}(t), t_{0}, t\right)$ satisfy:

(P1) $\left|L_{t} \cap \mathcal{V}_{\lambda, R}(t)\right| \lesssim R$ for any vortex line $L_{t}$ at time $t$,

(P2) $\left|\mathcal{V}_{\lambda, R}^{0}(t)\right| \lesssim \ell_{\lambda, R}^{0}(t) R^{d-2}$ where

$$
\ell_{\lambda, R}^{0}(t) \stackrel{\text { def }}{=} \sup _{L_{t_{0}} \subset \mathcal{T}\left(t_{0}\right)}\left|L_{t_{0}} \cap \mathcal{V}_{\lambda, R}^{0}(t)\right|
$$
and $\mathcal{T}\left(t_{0}\right)$ denotes the set of all vortex lines $L_{t_{0}}$ at time $t_{0}$.

Then, we get that for any $t \in\left[t_{0}, T^{*}[\right.$

$$
\left|\mathcal{V}_{\lambda, R}(t)\right| \lesssim\left\|\omega\left(t_{0}\right)\right\|_{\infty} \frac{R^{d-1}}{\lambda} .
$$


Proof. Let us take $t \in\left[t_{0}, T^{*}\left[\right.\right.$. If $\left|\mathcal{V}_{\lambda, R}(t)\right|=0$ the proof follows immediately. Then we assume that $\left|\mathcal{V}_{\lambda, R}(t)\right|>0$. We have $\left|\mathcal{V}_{\lambda, R}(t)\right|=\left|\mathcal{V}_{\lambda, R}^{0}(t)\right|$ since $X\left(\cdot, t_{0}, t\right)$ is a volume preserving $C^{1}$-diffeomorphism from $\mathbb{R}^{d}$ to $\mathbb{R}^{d}$, then we get $\left|\mathcal{V}_{\lambda, R}^{0}(t)\right|>0$ and Property (P2) yields to

$$
\left|\mathcal{V}_{\lambda, R}(t)\right| \lesssim \ell_{\lambda, R}^{0}(t) R^{d-2} .
$$

Furthermore for any vortex line $L_{t_{0}}$ at time $t_{0}$, we notice $X\left(L_{t_{0}} \cap \mathcal{V}_{\lambda, R}^{0}(t), t_{0}, t\right)=$ $L_{t} \cap \mathcal{V}_{\lambda, R}(t)$ where $L_{t}$ is the vortex line at time $t$ defined by $L_{t}=X\left(L_{t_{0}}, t_{0}, t\right)$. Then, for any vortex line $L_{t_{0}}$ at time $t_{0}$ such that $\left|L_{t_{0}} \cap \mathcal{V}_{\lambda, R}^{0}(t)\right|>0$, by using the equation just below of (3.12) in Deng et al. (2005) and the definition of the set $\mathcal{V}_{\lambda, R}(t)$, we obtain

$$
\frac{\left|L_{t} \cap \mathcal{V}_{\lambda, R}(t)\right|}{\left|L_{t_{0}} \cap \mathcal{V}_{\lambda, R}^{0}(t)\right|} \geq \frac{\lambda}{\left\|\omega\left(t_{0}\right)\right\|_{\infty}}
$$

${ }_{42}$ By using Property (P1), for any vortex line $L_{t_{0}}$ at time $t_{0}$ such that $\mid L_{t_{0}} \cap$ $\mathcal{V}_{\lambda, R}^{0}(t) \mid>0$, we deduce from (119) that $\frac{R}{\left|L_{t_{0}} \cap \mathcal{V}_{\lambda, R}^{0}(t)\right|} \gtrsim \frac{\lambda}{\left\|\omega\left(t_{0}\right)\right\|_{\infty}}$ which implies

$$
\frac{R}{\ell_{\lambda, R}^{0}(t)} \gtrsim \frac{\lambda}{\left\|\omega\left(t_{0}\right)\right\|_{\infty}} \text {. }
$$

Therefore by using (120), from (118) we thus infer $\left|\mathcal{V}_{\lambda, R}(t)\right| \lesssim\left\|\omega\left(t_{0}\right)\right\|_{\infty} \frac{R^{d-1}}{\lambda}$, which concludes the proof.

Thanks to assumption (116), we obtain the following Proposition 7.1.

Proposition 7.1. Let $d \in\{2,3\}, u_{0} \in H_{\sigma}^{r}\left(\mathbb{R}^{d}\right)$ with $r>\frac{d}{2}+3$. Let $T^{*}>0$ be such that there exists a unique strong solution $u$ to the $3 D$ Navier-Stokes, 3D Euler equations (9)-(10) or 2D QG equations (11)-(12) in the class

$$
u \in C\left(\left[0, T^{*}\left[; H_{\sigma}^{r}\left(\mathbb{R}^{d}\right)\right) \cap C^{1}\left(\left[0, T^{*}\left[; H^{r-2}\left(\mathbb{R}^{d}\right)\right) .\right.\right.\right.\right.
$$

Under the definitions (32)-(37) in the Theorem 5.1, we assume that there exists $t_{0} \in\left[0, T^{*}\left[\right.\right.$ such that for any $t \in\left[t_{0}, T^{*}[, x \in \Theta(t)\right.$, we get that for all $\lambda \geq \Omega(t)$ and $0<R \leq \rho_{0}(t)$

$$
|\{y \in B(x, R) ;|\omega(y, t)| \geq \lambda\}| \lesssim t_{0} \frac{R^{d-1}}{\lambda} .
$$

${ }_{54}$ Then we get that for all $t \in\left[t_{0}, T^{*}[\right.$

$$
\pi(t) \lesssim t_{0} 1+\log ^{+}\left(\frac{\|\omega(t)\|_{\infty}}{\Omega(t)}\right) .
$$


Proof. We have for all $t \in\left[t_{0}, T^{*}\left[, x \in \Theta(t)\right.\right.$ and $0<R \leq \rho_{0}(t)$

$$
\begin{aligned}
\int_{B(x, R) \cap \mathcal{V}(t)}|\omega(z, t)| d z & =\int_{B(x, R) \cap \mathcal{V}(t)} \int_{0}^{|\omega(z, t)|} d \lambda d z \\
& =\Omega(t)|B(x, R) \cap \mathcal{V}(t)|+\int_{B(x, R) \cap \mathcal{V}(t)} \int_{\Omega(t)}^{|\omega(z, t)|} d \lambda d z \\
& =\Omega(t)|B(x, R) \cap \mathcal{V}(t)|+\int_{\{z \in B(x, R), \Omega(t)<\lambda<|\omega(z, t)|\}} d \lambda d z \\
& =\Omega(t)|B(x, R) \cap \mathcal{V}(t)| \\
& +\int_{\left[\Omega(t),\|\omega(t)\|_{\infty}\right]}|\{z \in B(x, R) ;|\omega(z, t)|>\lambda\}| d \lambda,
\end{aligned}
$$

856 where we have used Fubini-Tonelli Theorem. Thanks to (121) we deduce that 857 for all $t \in\left[t_{0}, T^{*}\left[, x \in \Theta(t)\right.\right.$ and $0<R \leq \rho_{0}(t)$

$$
\begin{aligned}
\int_{B(x, R) \cap \mathcal{V}(t)}|\omega(z, t)| d z & \lesssim t_{0} R^{d-1}\left(1+\int_{\left[\Omega(t),\|\omega(t)\|_{\infty}\right]} \frac{d \lambda}{\lambda}\right) \\
& =R^{d-1}\left(1+\log ^{+}\left(\frac{\|\omega(t)\|_{\infty}}{\Omega(t)}\right)\right) .
\end{aligned}
$$

Owing to (122), we thus conclude the proof.

Remark 7.1. The analysis led in Kuznetsov and Ruban (2000); Kuznetsov et al. (2001) for the study of collapse of vortex lines and agrees with numerical experiments Agafontsev et al. $(2015,2017)$ suggests that the thickness of the regions of high vorticity $\{y \in B(x, R) ;|\omega(y, t)| \geq \lambda\}$ is $\frac{1}{\lambda^{\frac{3}{2}}}$ and since these regions are included in the ball $B(x, R)$, we expect that $|\{y \in B(x, R) ;|\omega(y, t)| \geq \lambda\}| \lesssim t_{0}$ $\frac{R^{d-1}}{\lambda^{\frac{3}{2}}}$. Then under this assumption and by using the same arguments as previously, we obtain

$$
\pi(t) \lesssim \Omega(t)^{-\frac{1}{2}}
$$

Thanks to Theorem 5.1 and Proposition 7.1 we obtain Theorem 7.1.

Theorem 7.1. Let $d \in\{2,3\}, u_{0} \in H_{\sigma}^{r}\left(\mathbb{R}^{d}\right)$ with $r>\frac{d}{2}+3$. Let $T^{*}>0$ be such that there exists a unique strong solution $u$ to the $3 D$ Navier-Stokes, $3 D$ Euler equations (9)-(10) or 2D QG equations (11)-(12) in the class

$$
u \in C\left(\left[0, T^{*}\left[; H_{\sigma}^{r}\left(\mathbb{R}^{d}\right)\right) \cap C^{1}\left(\left[0, T^{*}\left[; H^{r-2}\left(\mathbb{R}^{d}\right)\right) .\right.\right.\right.\right.
$$

Under the definitions (32)-(35) in the Theorem 5.1, we assume that there exists $t_{0} \in\left[0, T^{*}\left[\right.\right.$ such that for any $t \in\left[t_{0}, T^{*}[, x \in \Theta(t)\right.$, we get that for all $\lambda \geq \Omega(t)$ and $0<R \leq \rho_{0}(t)$

$$
|\{y \in B(x, R) ;|\omega(y, t)| \geq \lambda\}| \lesssim_{t_{0}} \frac{R^{d-1}}{\lambda} .
$$




$$
\int_{t_{1}}^{T^{*}} \mathbf{A}_{d}(t)\left(1+\log ^{+}\left(\frac{\|\omega(t)\|_{\infty}}{\Omega(t)}\right)\right) d t<+\infty,
$$

then the solution $u$ cannot blowup at the finite time $T^{*}$ where

$$
\begin{aligned}
& \mathbf{A}_{d}(t) \stackrel{\text { def }}{=} \sup _{x \in \Theta(t)} \sup _{y \in B(0, \rho(t)) \backslash\{0\}} \frac{\mathbf{D}_{d}(\hat{y}, \xi(x+y, t), \xi(x, t))^{+}}{|y|} \\
& \rho(t)=O\left(\left(T^{*}-t\right)\left(1+\log ^{+}\left(\frac{\|\omega(t)\|_{\infty}}{\Omega(t)}\right)\right)\right) .
\end{aligned}
$$

Remark 7.2. Under the considerations of Remark \%.1, the non blow-up of the solutions of Euler equations is obtained if there exists $t_{1} \in\left[t_{0}, T^{*}[\right.$ such that

$$
\int_{t_{1}}^{T^{*}} \mathbf{A}_{d}(t) \Omega(t)^{-\frac{1}{2}} d t<+\infty .
$$

Now, in the case of Euler equations and 2D QG equations by using their Lagrangian formulation, after a fine and sharp analysis of the expression of $\pi$ (37) we go further in the non blow-up criteria by showing in Proposition 7.2 under mild assumptions based on the anisotropic structure of regions of high vorticity, that $\pi(t)=O(1)$. For this purpose, we need the Lemmata 7.2, 7.3 and 7.4.

Lemma 7.2. Let $d \in\{2,3\}, u_{0} \in H_{\sigma}^{r}\left(\mathbb{R}^{d}\right)$ with $r>\frac{d}{2}+3$. Let $T^{*}>0$ be such that there exists a unique strong solution $u$ to the $3 D$ Navier-Stokes, $3 D$ Euler equations (9)-(10) or $2 D$ QG equations (11)-(12) in the class

$$
u \in C\left(\left[0, T^{*}\left[; H_{\sigma}^{r}\left(\mathbb{R}^{d}\right)\right) \cap C^{1}\left(\left[0, T^{*}\left[; H^{r-2}\left(\mathbb{R}^{d}\right)\right) .\right.\right.\right.\right.
$$

Let $\mathcal{A}_{0}$ be a smooth surface of $\mathbb{R}^{3}$ with boundary if $d=3$ or a curve of $\mathbb{R}^{2}$ if $d=2$. For any $t_{0} \in\left[0, T^{*}\left[\right.\right.$, let $\mathcal{A}\left(t_{0}, t\right) \stackrel{\text { def }}{=} X\left(\mathcal{A}_{0}, t_{0}, t\right)$ be the evolution of $\mathcal{A}_{0}$ through the flow map $X$ from the time $t_{0}$ to $t$, for any $t \in\left[0, T^{*}[\right.$. For any $t_{0} \in\left[0, T^{*}\left[\right.\right.$ and $t \in\left[0, T^{*}[\right.$, let

$$
\Gamma\left(t_{0}, t\right) \stackrel{\text { def }}{=} \int_{\mathcal{A}\left(t_{0}, t\right)}\left|\omega(y, t) \cdot \boldsymbol{n}_{t}(y)\right| d \sigma(y),
$$

where $\boldsymbol{n}_{t}(\cdot)$ denotes a unit normal vector of $\mathcal{A}\left(t_{0}, t\right)$.

Then for any $t_{0} \in\left[0, T^{*}\left[\right.\right.$ we get that $\Gamma\left(t_{0}, \cdot\right)$ is constant over $\left[0, T^{*}[\right.$, that is to say for all $t \in\left[t_{0}, T^{*}[\right.$,

$$
\Gamma\left(t_{0}, t\right)=\Gamma\left(t_{0}, t_{0}\right) .
$$

Proof. Thanks to Lemma 5 and Remark 3 of Schmidt and Schulz (2010) (see also (4.9) chapter 9 in Delfour and Zolésio (2011)) and Lemma 7 of Schmidt and Schulz (2010), we infer that for any $t_{0} \in\left[0, T^{*}\left[\right.\right.$ and $t \in\left[0, T^{*}[\right.$

$$
\int_{\mathcal{A}\left(t_{0}, t\right)}\left|\omega(y, t) \cdot \boldsymbol{n}_{t}(y)\right| d \sigma(y)=\int_{\mathcal{A}_{0}}\left|\omega\left(X\left(\alpha, t_{0}, t\right), t\right) \cdot\left(\nabla X\left(\alpha, t_{0}, t\right)\right)^{-T} \boldsymbol{n}(\alpha)\right| d \sigma(\alpha),
$$


where $\boldsymbol{n}$ is a unit normal vector of $\mathcal{A}_{0}$. Thanks to (113), we infer that for any $\alpha \in \mathcal{A}_{0}, t_{0} \in\left[0, T^{*}\left[\right.\right.$ and $t \in\left[0, T^{*}[\right.$

$$
\omega\left(X\left(\alpha, t_{0}, t\right), t\right) \cdot\left(\nabla X\left(\alpha, t_{0}, t\right)\right)^{-T} \boldsymbol{n}(\alpha)=\omega\left(\alpha, t_{0}\right) \cdot \boldsymbol{n}(\alpha) .
$$

Therefore, we thus obtain that for any $t_{0} \in\left[0, T^{*}\left[\right.\right.$ and $t \in\left[0, T^{*}[\right.$

$$
\int_{\mathcal{A}\left(t_{0}, t\right)}\left|\omega(y, t) \cdot \boldsymbol{n}_{t}(y)\right| d \sigma(y)=\int_{\mathcal{A}_{0}}\left|\omega\left(\alpha, t_{0}\right) \cdot \boldsymbol{n}(\alpha)\right| d \sigma(\alpha),
$$

which concludes the proof.

Lemma 7.3. Let $d \in\{2,3\}, u_{0} \in H_{\sigma}^{r}\left(\mathbb{R}^{d}\right)$ with $r>\frac{d}{2}+3$. Let $T^{*}>0$ be such that there exists a unique strong solution $u$ to the $3 D$ Navier-Stokes, 3D Euler equations (9)-(10) or $2 D Q G$ equations (11)-(12) in the class

$$
u \in C\left(\left[0, T^{*}\left[; H_{\sigma}^{r}\left(\mathbb{R}^{d}\right)\right) \cap C^{1}\left(\left[0, T^{*}\left[; H^{r-2}\left(\mathbb{R}^{d}\right)\right) .\right.\right.\right.\right.
$$

Let $t \in\left[0, T^{*}\left[\right.\right.$ and $\mathfrak{T}_{t}$ a vortex tube at this time. Let $\mathcal{A}_{t}$ and $\mathcal{B}_{t}$ be two connected smooth orientable surfaces of $\mathbb{R}^{3}$ (resp. curves of $\mathbb{R}^{2}$ if $d=2$ ) with boundary such their boundary encircle the vortex tube $\mathfrak{T}_{t}$ and such that any vortex line of the vortex tube $\mathfrak{T}_{t}$ intersects both $\mathcal{A}_{t}$ and $\mathcal{B}_{t}$ once each of them.

Then we get

$$
\int_{\mathcal{A}_{t}}\left|\omega(y, t) \cdot \boldsymbol{n}_{t}(y)\right| d \sigma(y)=\int_{\mathcal{B}_{t}}\left|\omega(y, t) \cdot \tilde{\boldsymbol{n}}_{t}(y)\right| d \tilde{\sigma}(y),
$$

where $\boldsymbol{n}_{t}$ and $\tilde{\boldsymbol{n}}_{t}$ are respectively the unit normal vector varying smoothly on the surfaces (resp. curves if $d=2$ ) $\mathcal{A}_{t}$ and $\mathcal{B}_{t}$, oriented to be outward to the portion of the tube $\mathfrak{T}_{t}$ delimited by $\mathcal{A}_{t}$ and $\mathcal{B}_{t}$.

Proof. For any $x \in \mathcal{A}_{t}$, we denote by $\mathfrak{T}_{t}(x)$ the vortex line passing through $x$ at time $t$, we get $\mathfrak{T}_{t}(x) \subset \mathfrak{T}_{t}$ and there exists an unique $y_{x, t} \in \mathcal{B}_{t}$ such that $\mathfrak{T}_{t}(x) \cap \mathcal{B}_{t}=\left\{y_{x, t}\right\}$. We thus introduce the function $\boldsymbol{\Phi}_{t}$ defined from $\mathcal{A}_{t}$ to $\mathcal{B}_{t}$ for all $x \in \mathcal{A}_{t}$ by $\boldsymbol{\Phi}_{t}(x)=y_{x, t}$. Since any vortex line of the vortex tube $\mathfrak{T}_{t}$ intersects both $\mathcal{A}_{t}$ and $\mathcal{B}_{t}$ once each of them and since also $\mathcal{A}_{t}$ and $\mathcal{B}_{t}$ are smooth surfaces (smooth curves if $d=2$ ), we infer thanks also to (115) that the function $\boldsymbol{\Phi}_{t}$ is a homeomorphism from $\mathcal{A}_{t}$ to $\mathcal{B}_{t}$.

We introduce the pairwise disjoints subsets of $\mathcal{A}_{t}$, namely $\mathcal{A}_{t}^{+} \stackrel{\text { def }}{=}\left\{y \in \mathcal{A}_{t} ; \omega(y, t)\right.$. $\left.\boldsymbol{n}_{t}(y)>0\right\}, \mathcal{A}_{t}^{-} \stackrel{\text { def }}{=}\left\{y \in \mathcal{A}_{t} ; \omega(y, t) \cdot \boldsymbol{n}_{t}(y)<0\right\}$ and $\mathcal{A}_{t}^{0} \stackrel{\text { def }}{=}\left\{y \in \mathcal{A}_{t} ; \omega(y, t)\right.$. $\left.\boldsymbol{n}_{t}(y)=0\right\}$. By the Sobolev embedding $H^{r-1}\left(\mathbb{R}^{d}\right) \hookrightarrow B C^{m_{r}}\left(\mathbb{R}^{d}\right), m_{r}=$ $\left[r-1-\frac{d}{2}\right] \geq 2$, we get $\omega(t) \in B C^{m_{r}}\left(\mathbb{R}^{d}\right)$. Then $\mathcal{A}_{t}^{+}$and $\mathcal{A}_{t}^{-}$are open subsets of the surface (curve if $d=2$ ) $\mathcal{A}_{t}$ and thus they are also smooth surfaces (smooth 


$$
\begin{aligned}
\int_{\mathcal{A}_{t}}\left|\omega(y, t) \cdot \boldsymbol{n}_{t}(y)\right| d \sigma(y) & =\int_{\mathcal{A}_{t}^{+}}\left|\omega(y, t) \cdot \boldsymbol{n}_{t}(y)\right| d \sigma(y)+\int_{\mathcal{A}_{t}^{-}}\left|\omega(y, t) \cdot \boldsymbol{n}_{t}(y)\right| d \sigma(y) \\
& +\int_{\mathcal{A}_{t}^{0}}\left|\omega(y, t) \cdot \boldsymbol{n}_{t}(y)\right| d \sigma(y) \\
& =\int_{\mathcal{A}_{t}^{+}} \omega(y, t) \cdot \boldsymbol{n}_{t}(y) d \sigma(y)-\int_{\mathcal{A}_{t}^{-}} \omega(y, t) \cdot \boldsymbol{n}_{t}(y) d \sigma(y),
\end{aligned}
$$

${ }_{924}$ where we have used the definition of the sets $\mathcal{A}_{t}^{+}, \mathcal{A}_{t}^{-}$and $\mathcal{A}_{t}^{0}$. Since $\boldsymbol{\Phi}_{t}$ is a ${ }_{925}$ homeomorphism from $\mathcal{A}_{t}$ to $\mathcal{B}_{t}$, we get that $\boldsymbol{\Phi}_{t}\left(\mathcal{A}_{t}^{+}\right) \subset \mathcal{B}_{t}, \boldsymbol{\Phi}_{t}\left(\mathcal{A}_{t}^{-}\right) \subset \mathcal{B}_{t}$ and ${ }_{926} \boldsymbol{\Phi}_{t}\left(\mathcal{A}_{t}^{+}\right) \cap \boldsymbol{\Phi}_{t}\left(\mathcal{A}_{t}^{-}\right)=\emptyset$. On the other hand, thanks to Helmholtz's first vortex ${ }_{927}$ Theorem (see e.g (Wu, 2018, chapter 2)), we have

$$
\int_{\mathcal{A}_{t}^{+}} \omega(y, t) \cdot \boldsymbol{n}_{t}(y) d \sigma(y)=-\int_{\boldsymbol{\Phi}_{t}\left(\mathcal{A}_{t}^{+}\right)} \omega(y, t) \cdot \tilde{\boldsymbol{n}}_{t}(y) d \tilde{\sigma}(y),
$$

928 and

$$
\int_{\mathcal{A}_{t}^{-}} \omega(y, t) \cdot \boldsymbol{n}_{t}(y) d \sigma(y)=-\int_{\boldsymbol{\Phi}_{t}\left(\mathcal{A}_{t}^{-}\right)} \omega(y, t) \cdot \tilde{\boldsymbol{n}}_{t}(y) d \tilde{\sigma}(y) .
$$

929 Then owing to (124) and (125), from (123) we deduce

$$
\begin{aligned}
\int_{\mathcal{A}_{t}}\left|\omega(y, t) \cdot \boldsymbol{n}_{t}(y)\right| d \sigma(y) & =-\int_{\Phi_{t}\left(\mathcal{A}_{t}^{+}\right)} \omega(y, t) \cdot \tilde{\boldsymbol{n}}_{t}(y) d \tilde{\sigma}(y) \\
& +\int_{\boldsymbol{\Phi}_{t}\left(\mathcal{A}_{t}^{-}\right)} \omega(y, t) \cdot \tilde{\boldsymbol{n}}_{t}(y) d \tilde{\sigma}(y),
\end{aligned}
$$

${ }_{930}$ which implies

$$
\int_{\mathcal{A}_{t}}\left|\omega(y, t) \cdot \boldsymbol{n}_{t}(y)\right| d \sigma(y) \leq \int_{\mathcal{B}_{t}}\left|\omega(y, t) \cdot \tilde{\boldsymbol{n}}_{t}(y)\right| d \tilde{\sigma}(y) .
$$

931 It remains to show that

$$
\int_{\mathcal{B}_{t}}\left|\omega(y, t) \cdot \tilde{\boldsymbol{n}}_{t}(y)\right| d \tilde{\sigma}(y) \leq \int_{\mathcal{A}_{t}}\left|\omega(y, t) \cdot \boldsymbol{n}_{t}(y)\right| d \sigma(y) .
$$

By introducing the pairwise disjoints subsets of $\mathcal{B}_{t}$, namely $\mathcal{B}_{t}^{+} \stackrel{\text { def }}{=}\left\{y \in \mathcal{B}_{t} ; \omega(y, t)\right.$. $\left.\tilde{\boldsymbol{n}}_{t}(y)>0\right\}, \mathcal{B}_{t}^{-} \stackrel{\text { def }}{=}\left\{y \in \mathcal{B}_{t} ; \omega(y, t) \cdot \tilde{\boldsymbol{n}}_{t}(y)<0\right\}$ and $\mathcal{B}_{t}^{0} \stackrel{\text { def }}{=}\left\{y \in \mathcal{B}_{t} ; \omega(y, t)\right.$. $\left.\tilde{\boldsymbol{n}}_{t}(y)=0\right\}$ and using the fact that $\boldsymbol{\Phi}_{t}^{-1}$ is a homeomorphism from $\mathcal{B}_{t}$ to $\mathcal{A}_{t}$, we deduce with the same arguments used to get (126), inequality (127). Then, owing to (126) and (127) we conclude the proof.

Before to turn to the proof of Lemma 7.4, Proposition 7.2 and Theorem 7.2, we need to introduce some definitions. Let $r>\frac{d}{2}+3$ and $T^{*}>0$ be such 
that there exists an unique strong solution $u$ to the 3D Navier-Stokes, 3D Euler equations (9)-(10) or 2D QG equations (11)-(12) in the class

$$
u \in C\left(\left[0, T^{*}\left[; H_{\sigma}^{r}\left(\mathbb{R}^{d}\right)\right) \cap C^{1}\left(\left[0, T^{*}\left[; H^{r-2}\left(\mathbb{R}^{d}\right)\right) .\right.\right.\right.\right.
$$

For any $t \in\left[0, T^{*}\left[\right.\right.$ and any vortex tube $\mathfrak{T}_{t}$ at time $t$, we define by

$\mathcal{S}\left(\mathfrak{T}_{t}\right)$ the set of the connected smooth orientable surfaces of $\mathbb{R}^{3}$ (curves of $\mathbb{R}^{2}$ if $d=2$ ) with boundary that is intersected only once by any vortex line of $\mathfrak{T}_{t}$ and such that their boundary encircle the vortex tube $\mathfrak{T}_{t}$.

We define also the function $\Gamma_{\mathfrak{T}_{t}}$ defined from $\mathcal{S}\left(\mathfrak{T}_{t}\right)$ to $\left[0,+\infty\left[\right.\right.$ for all $\mathcal{A} \in \mathcal{S}\left(\mathfrak{T}_{t}\right)$

$$
\Gamma_{\mathfrak{T}_{t}}(\mathcal{A}) \stackrel{\text { def }}{=} \int_{\mathcal{A}}|\omega(y, t) \cdot \boldsymbol{n}(y)| d \sigma(y) .
$$

Thanks to Lemma 7.3, we deduce that for any $t \in\left[0, T^{*}[\right.$ and any vortex tube $\mathfrak{T}_{t}$ at time $t$

$$
\Gamma_{\mathfrak{T}_{t}} \text { is constant over } \mathcal{S}\left(\mathfrak{T}_{t}\right) \text {. }
$$

Owing to (129), for any $t \in\left[0, T^{*}\left[\right.\right.$ and any vortex tube $\mathfrak{T}_{t}$ at time $t$, we define $\Gamma_{\text {abs }}\left(\mathfrak{T}_{t}\right)$ that we call the absolute strength of the vortex tube $\mathfrak{T}_{t}$ by

$$
\Gamma_{\mathrm{abs}}\left(\mathfrak{T}_{t}\right) \stackrel{\text { def }}{=} \Gamma_{\mathfrak{T}_{t}}\left(\mathcal{A}_{0}\right),
$$

with $\mathcal{A}_{0}$ an arbitrary element of $\mathcal{S}\left(\mathfrak{T}_{t}\right)$. As vortex tube moves with the fluid characterized by the flow map $X$ (thanks to Helmholtz's first vortex Theorem), then for any vortex tube $\mathfrak{T}_{t}$ at a time $t \in\left[0, T^{*}\left[\right.\right.$, we deduce that $X\left(\mathfrak{T}_{t}, t, \tau\right)$ is a vortex tube at time $\tau$ for any $\tau \in\left[0, T^{*}[\right.$.

Thanks to Lemma 7.2 , we infer that for any $t \in\left[0, T^{*}\left[\right.\right.$ and any vortex tube $\mathfrak{T}_{t}$ at time $t$,

$$
\boldsymbol{\Gamma}_{\text {abs }}\left(\mathfrak{T}_{t}\right)=\boldsymbol{\Gamma}_{\text {abs }}\left(X\left(\mathfrak{T}_{t}, t, \tau\right)\right) \text { for any } \tau \in\left[0, T^{*}[\text {, }\right.
$$

which means that the absolute strength of any vortex tube $\mathfrak{T}_{t}$ at a time $t \in$ $\left[0, T^{*}[\right.$ moving with the fluid does not change with the time.

Lemma 7.4. Let $d \in\{2,3\}, u_{0} \in H_{\sigma}^{r}\left(\mathbb{R}^{d}\right)$ with $r>\frac{d}{2}+3$. Let $T^{*}>0$ be such that there exists a unique strong solution $u$ to the $3 D$ Navier-Stokes, $3 D$ Euler equations (9)-(10) or $2 D Q G$ equations (11)-(12) in the class

$$
u \in C\left(\left[0, T^{*}\left[; H_{\sigma}^{r}\left(\mathbb{R}^{d}\right)\right) \cap C^{1}\left(\left[0, T^{*}\left[; H^{r-2}\left(\mathbb{R}^{d}\right)\right) .\right.\right.\right.\right.
$$

Let $t \in\left[0, T^{*}\left[\right.\right.$ and $\mathfrak{T}_{t}$ a vortex tube at time $t$ defined by $\mathfrak{T}_{t} \stackrel{\text { def }}{=}\left\{\mathbf{x}_{t}(\alpha, s) ; \alpha \in\right.$ $\left.\mathcal{A}_{t}, s \in J_{t}\right\}$ with $\mathcal{A}_{t}$ a connected smooth orientable surface of $\mathbb{R}^{3}$ (curve of $\mathbb{R}^{2}$ if $d=2$ ) with boundary and $J_{t}$ an interval of $\mathbb{R}$ containing 0 such that

$$
\text { - } J_{t} \subset \bigcap_{\alpha \in \mathcal{A}_{t}} \mathfrak{J}_{\alpha, t},
$$

- any vortex line of the tube $\mathfrak{T}_{t}$ intersects $\mathcal{A}_{t}$ only once, i.e

$$
\forall \beta \in \mathcal{A}_{t},\left\{\mathbf{x}_{t}(\beta, s) ; s \in \mathfrak{J}_{\beta, t}\right\} \cap \mathcal{A}_{t}=\{\beta\} .
$$


Then we have

$$
\int_{\mathfrak{T}_{t}}|\omega(z, t)| d z=\left|J_{t}\right| \boldsymbol{\Gamma}_{\mathrm{abs}}\left(\mathfrak{T}_{t}\right) .
$$

968 Proof. If $J_{t}=0$ then the result follows immediately. Therefore we assume that ${ }_{969} J_{t} \neq\{0\}$. For any $s \in J_{t}$, we define the smooth surface of $\mathbb{R}^{3}$ (curve of $\mathbb{R}^{2}$ if $970 \quad d=2$ ) with boundary,

$$
\mathcal{A}_{t}(s) \stackrel{\text { def }}{=}\left\{\mathbf{x}_{t}(\alpha, s) ; \alpha \in \mathcal{A}_{t}\right\} .
$$

${ }_{971}$ Due to the definition of the vortex tube $\mathfrak{T}_{t}$, we get that for any $s \in J_{t}$, the ${ }_{972}$ boundary of $\mathcal{A}_{t}(s)$ encircles the vortex tube $\mathfrak{T}_{t}$. Thanks to (114), we get

$$
\int_{\mathfrak{T}_{t}}|\omega(z, t)| d z=\int_{s \in J_{t}} \int_{\mathcal{A}_{t}(s)}|\omega(y, t)|\left|\boldsymbol{n}_{t}(s) \cdot \xi(y, t)\right| d \sigma(\alpha) d s,
$$

where $\boldsymbol{n}_{t}(s)$ is a unit normal vector of $\mathcal{A}_{t}(s)$. Since $\omega(y, t)=|\omega(y, t)| \xi(y, t)$ then we obtain

$$
\begin{aligned}
\int_{\mathfrak{T}_{t}}|\omega(z, t)| d z & =\int_{s \in J_{t}} \int_{\mathcal{A}_{t}(s)}\left|\omega(y, t) \cdot \boldsymbol{n}_{t}(s)\right| d \sigma(\alpha) d s \\
& =\int_{s \in J_{t}} \Gamma_{\mathfrak{T}_{t}}\left(\mathcal{A}_{t}(s)\right) d s .
\end{aligned}
$$

We show now that for any $s_{0} \in J_{t}$, any vortex line of the vortex tube $\mathfrak{T}_{t}$ intersects $\mathcal{A}_{t}\left(s_{0}\right)$ only once. For this purpose, let $\alpha_{1} \in \mathcal{A}_{t}\left(s_{0}\right)$. Thanks to Cauchy-Lipschitz Theorem (see e.g Theorem 2.2 in Teschl (2012)) used for (114), we deduce that there exists an unique $\beta_{1} \in \mathcal{A}_{t}$ such that $\alpha_{1}=\mathbf{x}_{t}\left(\beta_{1}, s_{0}\right)$. Suppose for a contradiction that

$$
\left\{\mathbf{x}_{t}\left(\beta_{1}, s\right) ; s \in \mathfrak{J}_{\beta_{1}, t}\right\} \cap \mathcal{A}_{t}\left(s_{0}\right) \neq\left\{\alpha_{1}\right\} .
$$

Then there exists $\alpha_{2} \neq \alpha_{1}$ such that $\alpha_{2} \in\left\{\mathbf{x}_{t}\left(\beta_{1}, s\right) ; s \in \mathfrak{J}_{\beta_{1}, t}\right\} \cap \mathcal{A}_{t}\left(s_{0}\right)$. Therefore we get that $\alpha_{2}=\mathbf{x}_{t}\left(\beta_{1}, s_{2}\right)$ with $s_{2} \in \mathfrak{J}_{\beta_{1}, t}, s_{2} \neq s_{0}$ since $\alpha_{2} \neq \alpha_{1}$. We get also that there exists an unique $\beta_{2} \in \mathcal{A}_{t}$ such that $\alpha_{2}=\mathbf{x}_{t}\left(\beta_{2}, s_{0}\right)$ where $\beta_{2} \neq \beta_{1}$ since $\alpha_{2} \neq \alpha_{1}$. We thus infer that

$$
\mathbf{x}_{t}\left(\beta_{1}, s_{2}\right)=\mathbf{x}_{t}\left(\beta_{2}, s_{0}\right) .
$$

By the maximality of $\mathbf{x}_{t}$, from (134) we infer that $s_{2}-s_{0} \in \mathfrak{J}_{\beta_{1}, t}$ and $\beta_{2}=$ $\mathbf{x}_{t}\left(\beta_{1}, s_{2}-s_{0}\right)$ which implies $\left\{\beta_{1}, \beta_{2}\right\} \subset\left\{\mathbf{x}_{t}\left(\beta_{1}, s\right) ; s \in \mathfrak{J}_{\beta_{1}, t}\right\} \cap \mathcal{A}_{t}$. This latter contradicts (132). Therefore, we deduce that

$$
\left\{\mathbf{x}_{t}\left(\beta_{1}, s\right) ; s \in \mathfrak{J}_{\beta_{1}, t}\right\} \cap \mathcal{A}_{t}\left(s_{0}\right)=\left\{\alpha_{1}\right\} .
$$

987 This means that the vortex line of the vortex tube $\mathfrak{T}_{t}$ passing through $\alpha_{1} \in$ $\mathcal{A}_{t}\left(s_{0}\right)$ intersects $\mathcal{A}_{t}\left(s_{0}\right)$ only once, which matches to our desired result. Then we get that for any $s \in J_{t}, \mathcal{A}_{t}(s) \in \mathcal{S}\left(\mathfrak{T}_{t}\right)$ and hence thanks to (129) and (130), from (133) we obtain $\int_{\mathfrak{T}_{t}}|\omega(z, t)| d z=\left|J_{t}\right| \boldsymbol{\Gamma}_{\text {abs }}\left(\mathfrak{T}_{t}\right)$. Then we conclude the proof. 
Proposition 7.2. Let $d \in\{2,3\}, u_{0} \in H_{\sigma}^{r}\left(\mathbb{R}^{d}\right)$ with $r>\frac{d}{2}+3$. Let $T^{*}>0$ be such that there exists a unique strong solution $u$ to the $3 D$ Navier-Stokes, 3D Euler equations (9)-(10) or 2D QG equations (11)-(12) in the class

$$
u \in C\left(\left[0, T^{*}\left[; H_{\sigma}^{r}\left(\mathbb{R}^{d}\right)\right) \cap C^{1}\left(\left[0, T^{*}\left[; H^{r-2}\left(\mathbb{R}^{d}\right)\right) .\right.\right.\right.\right.
$$

Under the definitions (32)-(37) in the Theorem 5.1, we assume that there exists $t_{0} \in\left[0, T^{*}\left[\right.\right.$ such that for any $t \in\left[t_{0}, T^{*}\left[, x \in \Theta(t)\right.\right.$ and $0<R \leq \rho_{0}(t)$ there exists a vortex tube $\mathfrak{T}_{x, t}^{R}$ defined by $\mathfrak{T}_{x, t}^{R} \stackrel{\text { def }}{=}\left\{\mathbf{x}_{t}(\alpha, s) ; \alpha \in \mathcal{A}_{x, t}^{R}, s \in I_{x, t}^{R}\right\}$ with $\mathcal{A}_{x, t}^{R}$ a connected smooth orientable surface of $\mathbb{R}^{3}$ (curve of $\mathbb{R}^{2}$ if $d=2$ ) and $I_{x, t}^{R}$ an interval of $\mathbb{R}$ containing 0 such that:

$(P 1) \mathcal{V}(t) \cap B(x, R) \subset \mathfrak{T}_{x, t}^{R}$.

(P2) any vortex line of the tube $\mathfrak{T}_{x, t}^{R}$ intersects $\mathcal{A}_{x, t}^{R}$ only once.

(P3) $\left|I_{x, t}^{R}\right| \lesssim R$

(P4) $\boldsymbol{\Gamma}_{\mathrm{abs}}\left(\mathfrak{T}_{x, t}^{R}\right) \lesssim \overline{\boldsymbol{v}}\left(t_{0}\right) R^{d-2}$ where $\overline{\boldsymbol{v}}\left(t_{0}\right)>0$ is a real which depend only on $t_{0}$ (and have the characteristic of a velocity).

Then we get that for all $t \in\left[t_{0}, T^{*}[\right.$

$$
\pi(t) \lesssim \overline{\boldsymbol{v}}\left(t_{0}\right)
$$

Proof. Let $t \in\left[t_{0}, T^{*}\left[, x \in \Theta(t)\right.\right.$ and $0<R \leq \rho_{0}(t)$. Thanks to property (P1) we have

$$
\int_{B(x, R) \cap \mathcal{V}(t)}|\omega(z, t)| d z \leq \int_{\mathfrak{T}_{x, t}^{R}}|\omega(z, t)| d z .
$$

Furthermore, thanks to property (P2) and Lemma 7.4 we get

$$
\int_{\mathfrak{T}_{x, t}^{R}}|\omega(z, t)| d z=\left|I_{x, t}^{R}\right| \boldsymbol{\Gamma}_{\mathrm{abs}}\left(\mathfrak{T}_{x, t}^{R}\right) .
$$

Thanks to the properties (P3) and (P4), from (136) we deduce

$$
\int_{\mathfrak{T}_{x, t}^{R}}|\omega(z, t)| d z \lesssim R^{d-1} \overline{\boldsymbol{v}}\left(t_{0}\right) .
$$

Owing to (137), from (135) we infer

$$
\int_{B(x, R) \cap \mathcal{V}(t)}|\omega(z, t)| d z \lesssim R^{d-1} \overline{\boldsymbol{v}}\left(t_{0}\right) .
$$

From the definition (37) of the function $\pi$, thanks to (138) we thus deduce that for all $t \in\left[t_{0}, T^{*}[\right.$

$$
\pi(t) \lesssim \overline{\boldsymbol{v}}\left(t_{0}\right)
$$

13 which concludes the proof. 
In the two following Remarks, we give explicit values for $\overline{\boldsymbol{v}}\left(t_{0}\right)$.

Remark 7.3. In the case of $2 D Q G$ equation for which $d=2$, we have that for all $t \in\left[t_{0}, T^{*}[\right.$,

$$
\pi(t) \lesssim\left\|u_{0}\right\|_{\infty}
$$

if we replace the hypothesis ( $\left.\mathrm{P}_{4}\right)$ by the assumption that the real-valued function $\omega(\cdot, t) \cdot \boldsymbol{n}$ keeps a constant sign over $\mathcal{A}_{x, t}^{R}$, where $\boldsymbol{n}$ is a unit normal vector varying smoothly on $\mathcal{A}_{x, t}^{R}$.

Indeed in this case, we get $\int_{\mathcal{A}_{x, t}^{R}}|\omega(\alpha, t) \cdot \boldsymbol{n}(\alpha)| d \alpha=\left|\int_{\mathcal{A}_{x, t}^{R}} \omega(\alpha, t) \cdot \boldsymbol{n}(\alpha) d \alpha\right|$ and furthermore thanks to Stokes Theorem we have $\int_{\mathcal{A}_{x+}^{R}} \omega(\alpha, t) \cdot \boldsymbol{n}(\alpha) d \alpha=$ $u\left(\alpha_{2}, t\right)-u\left(\alpha_{1}, t\right)$ where $\alpha_{2}$ and $\alpha_{1}$ are the two endpoints of the line segment $\mathcal{A}_{x, t}^{R}$. We thus infer $\int_{\mathcal{A}_{x, t}^{R}}|\omega(\alpha, t) \cdot \boldsymbol{n}(\alpha)| d \alpha \leq 2\|u(t)\|_{\infty}=2\left\|u_{0}\right\|_{\infty}$ thanks to (22) and then we take $\overline{\boldsymbol{v}}\left(t_{0}\right)=\left\|u_{0}\right\|_{\infty}$. Then with the properties (P1)-(P3), we thus obtain that for all $t \in\left[t_{0}, T^{*}\left[, \pi(t) \lesssim\left\|u_{0}\right\|_{\infty}\right.\right.$.

Remark 7.4. For any $t \in\left[t_{0}, T^{*}\left[, x \in \Theta(t)\right.\right.$ and $0<R \leq \rho_{0}(t)$ let us assume that there exists $t_{1} \in\left[0, t_{0}\right]$ depending on $t, x$ and $R$ such that for the vortex tube $\mathfrak{T}_{x, t_{1}}^{R} \stackrel{\text { def }}{=} X\left(\mathfrak{T}_{x, t}^{R}, t, t_{1}\right)$ at time $t_{1}$ we have

$$
\inf _{\mathcal{A} \in \mathcal{S}\left(\mathfrak{T}_{x, t_{1}}^{R}\right)}|\mathcal{A}| \lesssim R^{d-2},
$$

then Property (P4) holds with $\overline{\boldsymbol{v}}\left(t_{0}\right)=\|\omega\|_{L^{\infty}\left(\mathbb{R}^{d} \times\left[0, t_{0}\right]\right)}$. Indeed thanks to (131) we have

$$
\Gamma_{\text {abs }}\left(\mathfrak{T}_{x, t}^{R}\right)=\Gamma_{\text {abs }}\left(\mathfrak{T}_{x, t_{1}}^{R}\right) .
$$

Furthermore, thanks to (129) and (130), we deduce that

$$
\begin{aligned}
\Gamma_{\text {abs }}\left(\mathfrak{T}_{x, t_{1}}^{R}\right) & =\inf _{\mathcal{A} \in \mathcal{S}\left(\mathfrak{T}_{x, t_{1}}^{R}\right)} \int_{\mathcal{A}}\left|\omega\left(y, t_{1}\right) \cdot \boldsymbol{n}(y)\right| d \sigma(y) \\
& \leq\left\|\omega\left(t_{1}\right)\right\|_{\infty} \inf _{\mathcal{A} \in \mathcal{S}\left(\mathfrak{F}_{x, t_{1}}^{R}\right)}|\mathcal{A}| \\
& \lesssim\|\omega\|_{L^{\infty}\left(\mathbb{R}^{d} \times\left[0, t_{0}\right]\right)} R^{d-2} .
\end{aligned}
$$

Therefore, we deduce that $\boldsymbol{\Gamma}_{\mathrm{abs}}\left(\mathfrak{T}_{x, t}^{R}\right) \lesssim\|\omega\|_{L^{\infty}\left(\mathbb{R}^{d} \times\left[0, t_{0}\right]\right)} R^{d-2}$ which matches with Property (P4) for $\overline{\boldsymbol{v}}\left(t_{0}\right)=\|\omega\|_{L^{\infty}\left(\mathbb{R}^{d} \times\left[0, t_{0}\right]\right)}$.

Then thanks to Theorem 5.1 and Proposition 7.2 we deduce Theorem 7.2.

Theorem 7.2. Let $d \in\{2,3\}, u_{0} \in H_{\sigma}^{r}\left(\mathbb{R}^{d}\right)$ with $r>\frac{d}{2}+3$. Let $T^{*}>0$ be such that there exists a unique strong solution $u$ to the $3 D$ Navier-Stokes, $3 D$ Euler equations (9)-(10) or 2D QG equations (11)-(12) in the class

$$
u \in C\left(\left[0, T^{*}\left[; H_{\sigma}^{r}\left(\mathbb{R}^{d}\right)\right) \cap C^{1}\left(\left[0, T^{*}\left[; H^{r-2}\left(\mathbb{R}^{d}\right)\right) .\right.\right.\right.\right.
$$


Under the definitions (32)-(36) in the Theorem 5.1, we assume that there exists $t_{0} \in\left[0, T^{*}\left[\right.\right.$ such that for any $t \in\left[t_{0}, T^{*}\left[, x \in \Theta(t)\right.\right.$ and $0<R \leq \rho_{0}(t)$ there exists a vortex tube $\mathfrak{T}_{x, t}^{R}$ defined by $\mathfrak{T}_{x, t}^{R} \stackrel{\text { def }}{=}\left\{\mathbf{x}_{t}(\alpha, s) ; \alpha \in \mathcal{A}_{x, t}^{R}, s \in I_{x, t}^{R}\right\}$ with $\mathcal{A}_{x, t}^{R}$ a connected smooth orientable surface of $\mathbb{R}^{3}$ (curve of $\mathbb{R}^{2}$ if $d=2$ ) and $I_{x, t}^{R}$ an interval of $\mathbb{R}$ containing 0 such that:

$(P 1) \mathcal{V}(t) \cap B(x, R) \subset \mathfrak{T}_{x, t}^{R}$.

(P2) $\left|I_{x, t}^{R}\right| \lesssim R$

(P3) any vortex line of the tube $\mathfrak{T}_{x, t}^{R}$ intersects $\mathcal{A}_{x, t}^{R}$ only once.

(P4) $\boldsymbol{\Gamma}_{\mathrm{abs}}\left(\mathfrak{T}_{x, t}^{R}\right) \lesssim \overline{\boldsymbol{v}}\left(t_{0}\right) R^{d-2}$ where $\overline{\boldsymbol{v}}\left(t_{0}\right)>0$ is a real depending only on $t_{0}$ (and have the characteristic of a velocity).

Then if there exists $t_{1} \in\left[t_{0}, T^{*}[\right.$ such that

$$
\int_{t_{1}}^{T^{*}} \mathbf{A}_{d}(t) d t<+\infty
$$

then the solution $u$ cannot blowup at the finite time $T^{*}$ with

$$
\begin{aligned}
& \mathbf{A}_{d}(t) \stackrel{\text { def }}{=} \sup _{x \in \Theta(t)} \sup _{y \in B(0, \rho(t)) \backslash\{0\}} \frac{\mathbf{D}_{d}(\hat{y}, \xi(x+y, t), \xi(x, t))^{+}}{|y|} \\
& \rho(t)=O\left(\left(T^{*}-t\right) \overline{\boldsymbol{v}}\left(t_{0}\right)\right) .
\end{aligned}
$$

\section{References}

G. Luo and T. Y. Hou. Potentially singular solutions of the 3d axisymmetric euler equations. Proc. Natl. Acad. Sci., 111(36):12968-12973, 2014a.

G. Luo and T. Y. Hou. Toward the finite time blowup of the $3 \mathrm{~d}$ axisymmetric euler equations: A numerical investigation. Multiscale Model. Simul., 12(4): 1722-1776, 2014b.

T. Tao. Finite time blow-up for an averaged three-dimensional navier-stokes equations. J. Amer. Math. Soc., 29:601-674, 2016a.

T. Tao. Finite time blowup for lagrangian modifications of the three-dimensional euler equation. Ann. PDE, 2(9), 2016b.

M. P. Brenner, S. Hormoz, and A. Pumir. Potential singularity mechanism for the euler equations. Phys. Rev. Fluids, 1(084503), 2016.

P. Constantin. On the euler equations of incompressible fluids. Bull. Amer. Math. Soc., 44:603-621, 2007.

C. Bardos and E. S. Titi. Euler equations for an ideal incompressible fluid. Uspekhi Mat. Nauk, 62(3):5-46, 2007. 
J. Leray. Sur le mouvement d'un liquide visqueux emplissant l'espace. Acta Math., 63:193-248, 1934.

E. Hopf. über die anfangwertaufgabe für die hydrohynamischen grundgleichungen. Math. Nachr, 4:213-231, 1951.

O. Ladyzhenskaya. The Mathematical Theory of Viscous Incompressible Flows. Gordon and Breach, 2 edition, 1969.

J. L. Lions and G. Prodi. Un théorème d'existence et d'unicité dans les équations de navier-stokes en dimension 2. C. R. Acad. Sci. Paris, 248:3519-3521, 1959.

J. L. Lions. Quelques Méthodes de Résolution des Problèmes aux Limites non Linéaires. Dunod, Paris, 1969.

R. Temam. Navier-Stokes equations. North-Holland, Amsterdam, 1977.

G. Furioli, P. G. Lemarié-Rieusset, and E. Terraneo. Unicité dans $l^{3}\left(\mathbb{R}^{3}\right)$ et d'autres espaces fonctionnels limites pour navier-stokes. Rev. Mat. Iberoam., 16(3), 2000.

Y. Giga. Weak and strong solutions of the navier-stokes initial value problem. RIMS, Kyoto Univ, 19:887-910, 1983.

S. Monniaux. Unicité dans $l^{d}$ des solutions du système de navier-stokes : cas des domaines lipschitziens. Ann. Math. Blaise Pascal, 10:107-116, 2000.

J. L. Lions. Sur la régularité et l'unicité des solutions turbulentes des équations de navier-stokes. Rend. Semin. Mat. Univ. Padova, 30:16-23, 1960.

I. Gallagher and F. Planchon. On global infinite energy solutions to the navierstokes equations in two dimensions. Arch. Ration. Mech. Anal., 161:307-337, 2002.

J. Serrin. On the interior regularity of weak solutions of the navier-stokes equations. Arch. Ration. Mech. Anal., 9:187-191, 1962.

W. Von Wahl. Regularity of weak solutions of the navier-stokes equations. In The title of the book, pages 497-503. 1983 Summer Institute on Nonlinear Functional Analysis and Applications, Proc. Symposia in Pure Mathematics 45, Providence Rhode Island : Amer. Math. Soc., 1986.

Y. Giga. Solutions for semilinear parabolic equations in $l^{q}$ and regularity of weak solutions of the navier-stokes system. J. Differ. Equations, 62:186-212, 1986 .

L. Iskauriaza, G. A. Serëgin, and V. Shverak. $l_{3, \infty}$-solutions of navier-stokes equations and backward uniqueness. Uspekhi Mat. Nauk, 58(2):3-44, 2003.

C. He. Regularity for solutions to the navier-stokes equations with one velocity component regular. Electron. J. Differential Equations, 29:1-13, 2002. 
J. G. Heywood. Epochs of regularity for weak solutions of the navier-stokes equations in unbounded domains. Tôhoku Math. J., 40:293-313, 1988.

T. Kato. Strong $l^{q}$ solutions of the navier-stokes equations in $\mathbb{R}^{m}$, with application to weak solutions. Math. Z., 187:471-480, 1984.

T. Kato. Liapunov functions and monotonicity in the navier-stokes equations. Lecture Notes in Math., 1450:53-63, 1990.

H. Beirão da Veiga. A new regularity class for the navier-stokes equations in $\mathbb{R}^{n}$. Chin. Ann. Math. Ser. B, 16(4):407-412, 1995.

D. Chae and H-J. Choe. Regularity of solutions to the navier-stokes equations. Electron. J. Differential Equations, 5:1-7, 1999.

Y. Zhou. A new regularity criterion for the navier-stokes equations in terms of the gradient of one velocity component. Methods Appl. Anal., 9(4):563-578, 2002 .

P. Constantin and C. Fefferman. Direction of vorticity and the problem of global regularity for the navier-stokes equations. Indiana Univ. Math. J., 42: 775-789, 1994.

V. I. Yudovich. Non-stationary flows of an ideal incompressible fluid. Z. Vychisl. Mat. i Mat. Fiz., 6(3):1032-1066, 1963.

V. I. Yudovich. Uniqueness theorem for the basic nonstationary problem in the dynamics of an ideal incompressible fluid. Math. Res. Lett., 2(1):27-38, 1995.

M. Vishik. Incompressible flows of an ideal fluid with vorticity in borderline spaces of besov type. Ann. Sci. Éc. Norm. Supér., 32(6):769-812, 1999.

R. J. DiPerna and A. J. Majda. Diperna, r. j. and majda, a. j. Comm. Math. Phys., 108(4):667-689, 1987.

C. Camillo De Lellis and Jr. L. Székelyhidi. The h-principle and the equations of fluid dynamics. Bull. Amer. Math. Soc., 49(3):347-375, 2012.

C. Villani. Paradoxe de scheffer-shnirelman revu sous l'angle de l'intégration convexe [d'après c. de lellis et l. székelyhidi]. Séminaire Bourbaki $61^{e}$ année, no 1001, 2008-2009.

J. T. Beale, T. Kato, and A. Majda. Remarks on the breakdown of smooth solutions for the 3-d euler equations. Comm. Math. Phys., 94(1):61-66, 1984.

G. Ponce. Remarks on a paper by j. t. beale, t. kato, and a. majda. Comm. Math. Phys., 98:349-353, 1985.

A. B. Ferrari. On the blow-up of solutions of the 3 -d euler equations in a bounded domain. Comm. Math. Phys., 155:277-294, 1993. 
T. Shirota and T. Yanagisawa. A continuation principle for the 3-d euler equations for incompressible fluids in a bounded domain. Proc. Japan Acad. Ser. A Math. Sci., 69:77-82, 1993.

P. Constantin, C. Fefferman, and A. J. Majda. Geometric constraints on potentially singular solutions for the 3 -d euler equation. Comm. Partial Differential Equations, 21:559-571, 1996.

J. Deng, T. Y. Hou, and X. Yu. Geometric properties and non-blow-up of 3-d incompressible euler flow. Comm. Partial Differential Equations, 30(1): 225-243, 2005.

J. D. Gibbon and E. S. Titi. The 3d incompressible euler equations with a passive scalar: A road to blow-up ? J. Nonlinear Sci., 23:993-1000, 2013.

I.M. Held, R.T. Pierrehumbert, S.T. Harner, and K.L. Swanson. Surface quasigeostrophic dynamics. J. Fluid Mech., 282:1-20, 1995.

J. Pedlosky. Geophysical Fluid Dynamics. Springer, New York, 1987.

P. Constantin, A. Majda, and E. Tabak. Formation of strong fronts in the $2 \mathrm{~d}$ quasi-geostrophic thermal active scalar. Nonlinearity, 7:1495-1533, 1994.

D. Córdoba. Nonexistence of simple hyperbolic blow-up for the quasigeostrophic equation. Ann. of Math., 148:1135-1152, 1998.

D. Córdoba and C. Fefferman. Growth of solutions for qg and 2d euler equations. J. Amer. Math. Soc., 15:665-670, 2002.

D. Chae. The quasi-geostrophic equation in the triebel-lizorkin spaces. Nonlinearity, 16:479-495, 2003.

K. Ohkitani and M. Yamada. Inviscid and inviscid-limit behavior of a surface quasi- geostrophic flow. Phys. Fluids, 9(4):876-882, 1997.

P. Constantin, M-C. Lai, R. Sharma, Y-H. Tseng, and J. Wu. New numerical results for the surface quasi-geostrophic equation. J. Sci. Comput., 50(1): $1-28,2012$.

D. Chae, P. Constantin, and J. Wu. Deformation and symmetry in the inviscid sqg and the 3d euler equations. J. Nonlinear Sci., 22(5):665-688, 2012.

S. Montgomery-Smith. Finite time blow up for a navier-stokes like equation. Proc. Amer. Math. Soc., 129(10):3025-3029, 2001.

I. Gallagher and M. Paicu. Remarks on the blow-up of solutions to a toy model for the navier-stokes equations. Proc. Amer. Math. Soc., 137(6):2075-2083, 2009 .

D. Li and Ya. Sinai. Blow ups of complex solutions of the 3d-navier-stokes system and renormalization group method. J. Eur. Math. Soc., 10(2):267$313,2008$. 
P. Plecháç and V. Sverák. Singular and regular solutions of a nonlinear parabolic system. 16(6):2083-2097, 2003.

N. H. Katz and N. Pavlovic. Finite time blow-up for a dyadic model of the euler equations. Trans. Am. Math. Soc., 357(2):695-708, 2005.

T. Kambe. Gauge principle and variational formulation for flows of an ideal fluid. Acta Mech. Sin., 19(5):437-452, 2003a.

T. Kambe. Gauge principle for flows of an ideal fluid. Fluid Dyn. Res., 32: 193-199, 2003b.

P. Constantin. Geometric statistic in turbulence. SIAM Rev., 36(1):73-98, 1994.

H. Beirão da Veiga and L. C. Berselli. Navier-stokes equations: Green's matrices, vorticity direction, and regularity up to the boundary. J. Differ. Equations, 246:597-628, 2009.

L.C. Berselli. Some geometric constraints and the problem of global regularity for the navier-stokes equations. Nonlinearity, 22(10):2561-2581, 2009.

T. Y. Hou and C. Li. Dynamic stability of the 3d axi-symmetric navier-stokes equations with swirl. Commun. Pure Appl. Math., 61:661-697, 2008.

J. Deng, T. Y. Hou, and X. Yu. Improved geometric conditions for non-blow-up of 3d incompressible euler equation. Comm. Partial Differential Equations, 31:293-306, 2006a.

J. Deng, T. Y. Hou, R. Li, and X. Yu. Level set dynamics and the non-blow-up of the 2d quasi-geostrophic equation. Methods Appl. Anal., 13(2):157-180, 2006b.

R. M. Kerr and M. D. Bustamante. Exploring symmetry plane conditions in numerical euler solutions. In J. C. Robinson, J. L. JRodrigo, and W. Sadowski, editors, Mathematical Aspects of Fluid Mechanics. Cambridge University Press, 2012.

T. Grafke. Finite-time Euler singularities: A Lagrangian perspective. PhD thesis, 2012. PhD Thesis in der Fakultät für Physik und Astronomie der Ruhr-Universität Bochum.

B. N. Pshenichny. Necessary Conditions for an Extremum. Marcel Dekker, Inc.,New York, NY, 1971. Translated from Russian 1969.

R. M. Kerr. Velocity and scaling of collapsing euler vortices. Phys. Fluids, 14 (075103), 2005.

R. M. Kerr. The outer regions in singular euler. In Tsinober and eds. Gyr, editors, Fundamental Problematic Issues in Turbulence. Boston: Birkhäuser, 1998. 
R. M. Kerr. Euler singularities and turbulence. In 19th ICTAM Kyoto '96, Elsevier Science, 1997.

E. A. Kuznetsov and V. P. Ruban. Collapse of vortex lines in hydrodynamics. J. Exp. Theor. Phys., 91(4):775-785, 2000.

E. A. Kuznetsov, O. M. Podvigina, and V. A. Zheligovsky. Numerical evidence of breaking of vortex lines in an ideal fluid. In K. Bajer and H. K. Moffatt, editors, Tubes, Sheets and Singularities in Fluid Dynamics, pages 305-316. NATO ARW, Kluwer Academic Publishers, 2001.

D. S. Agafontsev, E. A. Kuznetsov, and A. A. Mailybaev. Development of high vorticity structures in incompressible 3d euler equations. Phys. Fluids, 27 (085102), 2015.

D. S. Agafontsev, E. A. Kuznetsov, and A. A. Mailybaev. Asymptotic solution for high-vorticity regions in incompressible three-dimensional euler equations. J. Fluid Mech., 813, 2017.

T. Grafke and R. Grauer. Finite-time euler singularities: A lagrangian perspective. Appl. Math. Lett., 26:500-505, 2013.

E. Stein. Singular Integrals and Differentiability Properties of Functions. Princeton NJ: Princeton University Press, 1970.

T. Kato and G. Ponce. Commutator estimates and the euler and navier-stokes equa- tions. Commun. Pure Appl. Math., 41(7):891-907, 1988.

J.P Bourguignon and H. Brezis. Remarks on the euler equation. J. Funct. Anal., 15:341-363, 1974.

T. Kato and G. Ponce. Well-posedness of the euler and navier-stokes equations in the lebesgue spaces $l_{s}^{p}\left(\backslash^{2}\right)$. Rev. Mat. Iberoam., 2:73-88, 1986.

H. Kozono and Y. Taniuchi. Bilinear estimates and critical sobolev inequality in bmo, with applications to the navier-stokes and the euler equations. RIMS Kokyuroku, 1146:39-52, 2000.

L. Agélas. Global regularity for logarithmically critical 2d mhd equations with zero viscosity. Monatsh. Math., 181(2):245-266, 2016.

D. Cordoba and C. Fefferman. On the collapse of tubes carried by 3d incompressible flows. Comm. Math. Phys., 222:293-298, 2001.

R. M. Kerr. Evidence for a singularity of the three-dimensional incompressible euler equations. Phys. Fluids A, 5:1725-1746, 1993.

R. M. Kerr. The role of singularities in Euler. In Small-Scale Structure in Hydro and Magnetohydrodynamic Turbulence. Springer-Verlag, pouquet, a., sulem, p. l., eds. lecture notes. edition, 1995. 
R. B. Pelz. Locally self-similar, finite-time collapse in a high-symmetry vortex filament model. Phys. Rev. E, 55(2):1617-1620, 1997.

R. B. Pelz. Symmetry and the hydrodynamic blow-up problem. J. Fluid Mech., 444:299-320, 2001.

O. F. Borisenko and L. I. Minchenko. Directional derivatives of the maximum function. Cybernet. Systems Anal., 28(2):309-312, 1992.

A. J. Chorin and J. E. Marsden. A Mathematical Introduction to Fluid Mechanics. 3rd ed. New York: Springer-Verlag, 1993.

M.V. Melander and F. Hussain. Cross-linking of two antiparallel vortex tubes. Phys. Fluids, 1:633-636, 1989.

R.M. Kerr and F. Hussain. Simulation of vortex reconnection. Phys. D, 37: 474-484, 1989.

A. Pumir and E. D. Siggia. Collapsing solutions to the 3-d euler equations. Phys. Fluids A, 2:220-241, 1990.

R. Grauer, C. Marliani, and K. Germaschewski. Adaptive mesh refinement for singular solutions of the incompressible euler equations. Phys. Rev. Fluids, 80:4177-4180, 1998.

T. Y. Hou and R. Li. Dynamic depletion of vortex stretching and non-blow-up of the 3-d incompressible euler equations. J. Nonlinear Sci., 16:639-664, 2006.

R. M. Kerr. Computational euler history, 2006.

S. Hormoz and M. P. Brenner. Absence of singular stretching of interacting vortex filaments. J. Fluid Mech., 707:191-204, 2012.

A. Majda and A. Bertozzi. Vorticity and Incompressible Flow. Cambridge Univ. Press., 2002.

G. Teschl. Ordinary Differential Equations and Dynamical Systems, volume 140 of Graduate Studies in Mathematics. Amer. Math. Soc., 2012.

S. Schmidt and V. Schulz. Shape derivatives for general objective functions and the incompressible navier-stokes equations. Control and Cybernetics, 39(3), 2010 .

M.C. Delfour and J.-P. Zolésio. Shapes and Geometries: Metrics, Analysis, Differential Calculus, and Optimization, Second Edition. Siam, 2011.

J. C. Wu. Elements of Vorticity Aerodynamics, volume 140 of Springer Tracts in Mechanical Engineering. Springer-Verlag Berlin Heidelberg, 2018. doi: 10.1007/978-3-662-44040-7. 